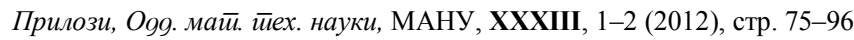
Contributions, Sec. Math. Tech. Sci., MANU, XXXIII, 1-2 (2012), pp. 75-96

ISSN $0351-3246$

UDC: $517.925: 512.622 .86$

\title{
SYSTEMS OF DIFFERENCE EQUATIONS APPROXIMATING THE LORENZ SYSTEM OF DIFFERENTIAL EQUATIONS
}

\author{
Biljana Zlatanovska, Dončo Dimovski
}

\begin{abstract}
A b s t r a c t: In this paper, starting from the Lorenz system of differential equations, some systems of difference equations are produced. Using some regularities in these systems of difference equations, polynomial approximations of their solutions are found. Taking these approximations as coefficients, three power series are obtained and by computer calculations is examined that these power series are local approximations of the solutions of the starting Lorentz system of differential equations.
\end{abstract}

Key words: Lorenz system; differential equations; difference equations; power series; approximation

\section{INTRODUCTION}

The use of power series is one of the oldest methods for examining differential equations. In the literature there are numerous papers concerned with such a use of power series, like the papers [1], [2] and [3].

We consider the well known Lorenz system of differential equations:

$$
\begin{aligned}
& \dot{x}=\sigma(y-x) \\
& \dot{y}=x(r-z)-y \\
& \dot{z}=x y-b z
\end{aligned}
$$

with three parameters $\sigma, r, b$.

For initial values $a_{0}=x(0), b_{0}=y(0), c_{0}=z(0)$, we assume that the solutions of the Lorenz system are expanded as Maclaurin series, (1.2). 


$$
\begin{aligned}
& x(t)=a_{0}+a_{1} t+a_{2} \frac{t^{2}}{2 !}+\ldots+a_{n} \frac{t^{n}}{n !}+\ldots=\sum_{n=0}^{\infty} a_{n} \frac{t^{n}}{n !} \\
& y(t)=b_{0}+b_{1} t+b_{2} \frac{t^{2}}{2 !}+\ldots+b_{n} \frac{t^{n}}{n !}+\ldots=\sum_{n=0}^{\infty} b_{n} \frac{t^{n}}{n !} \\
& z(t)=c_{0}+c_{1} t+c_{2} \frac{t^{2}}{2 !}+\ldots+c_{n} \frac{t^{n}}{n !}+\ldots=\sum_{n=0}^{\infty} c_{n} \frac{t^{n}}{n !}
\end{aligned}
$$

Using consecutive differentiation of (1.1) and the representation (1.2), for every $n \in N$, we obtain the following system of difference equations.

$$
\begin{aligned}
& a_{n}=\sigma\left(b_{n-1}-a_{n-1}\right) \\
& b_{n}=r a_{n-1}-b_{n-1}-\sum_{i=0}^{n-1}\left(\begin{array}{c}
n-1 \\
i
\end{array}\right) a_{i} c_{n-i-1} . \\
& c_{n}=-b c_{n-1}+\sum_{i=0}^{n-1}\left(\begin{array}{c}
n-1 \\
i
\end{array}\right) a_{i} b_{n-i-1}
\end{aligned}
$$

Our aim is to express the coefficients $a_{n}, b_{n}$ and $c_{n}$, as polynomials in variables $\sigma, r, b, a_{0}, b_{0}$ and $c_{0}$. Separately, for each of the coefficients $a_{n}, b_{n}$ and $c_{n}$ we transform the system (1.3), by introducing new variables.

\section{THE COEFFICIENT $a_{n}$}

We write the system (1.3) in the form (2.1).

$$
\begin{aligned}
& a_{n}=\sigma\left(b_{n-1}-a_{n-1}\right) \\
& b_{n}=r a_{n-1}-b_{n-1}-a_{0} c_{n-1}-\sum_{i=1}^{n-1}\left(\begin{array}{c}
n-1 \\
i
\end{array}\right) a_{i} c_{n-1-i} . \\
& c_{n}=a_{0} b_{n-1}-b c_{n-1}+\sum_{i=1}^{n-1}\left(\begin{array}{c}
n-1 \\
i
\end{array}\right) a_{i} b_{n-1-i}
\end{aligned}
$$

For a fixed $n \in N$ and for any $1 \leq k \leq n$ we represent the coefficients $a_{n}$ as:

$$
\begin{aligned}
a_{n}= & \varphi_{k}^{n} a_{n-k}+\psi_{k}^{n} b_{n-k}+\xi_{k}^{n} c_{n-k}+\sum_{m=0}^{k-1} \sum_{i=k-m}^{n-m-1} \tau_{k}^{n}(i, m) a_{i} b_{n-i-m-1}, \\
& +\sum_{m=0}^{k-1} \sum_{i=k-m}^{n-m-1} \pi_{k}^{n}(i, m) a_{i} c_{n-i-m-1}
\end{aligned}
$$


where $\varphi_{k}^{n}, \psi_{k}^{n}, \xi_{k}^{n}, \tau_{k}^{n}(i . m), \pi_{k}^{n}(i, m)$ are new variables. For the new variables we obtain the new systems of difference equations:

$$
\begin{aligned}
& \varphi_{k}^{n}=-\sigma \varphi_{k-1}^{n}+r \psi_{k-1}^{n} \\
& \psi_{k}^{n}=\sigma \varphi_{k-1}^{n}-\psi_{k-1}^{n}+a_{0} \xi_{k-1}^{n}+\sum_{i=1}^{k-1} a_{i}\left(\begin{array}{c}
n-k+i \\
i
\end{array}\right) \xi_{k-1-i}^{n} \\
& \xi_{k}^{n}=-a_{0} \psi_{k-1}^{n}-b \xi_{k-1}^{n}-\sum_{i=1}^{k-1} a_{i}\left(\begin{array}{c}
n-k+i \\
i
\end{array}\right) \psi_{k-1-i}^{n} \\
& \tau_{k}^{n}(i, m)=\xi_{m}^{n}\left(\begin{array}{c}
n-m-1 \\
i
\end{array}\right), \quad \pi_{k}^{n}(i, m)=-\psi_{m}^{n}\left(\begin{array}{c}
n-m-1 \\
i
\end{array}\right)
\end{aligned}
$$

with initial values $\varphi_{0}^{n}=1, \psi_{0}^{n}=0, \xi_{0}^{n}=0$, and transform the presentation of $a_{n}$ to

$$
\begin{aligned}
a_{n}= & \varphi_{k}^{n} a_{n-k}+\psi_{k}^{n} b_{n-k}+\xi_{k}^{n} c_{n-k}+\sum_{m=0}^{k-1} \sum_{i=k-m}^{n-m-1}\left(\begin{array}{c}
n-1-m \\
i
\end{array}\right) \xi_{m}^{n} a_{i} b_{n-i-m-1} \\
& -\sum_{m=0}^{k-1} \sum_{i=k-m}^{n-m-1}\left(\begin{array}{c}
n-1-m \\
i
\end{array}\right) \psi_{m}^{n} a_{i} c_{n-i-m-1} .
\end{aligned}
$$

For $n=k$, directly from the presentation of $a_{n}$, the inequality $n-m-1<n-m$, implies:

\section{1. $a_{n}=\varphi_{n}^{n} a_{0}+\psi_{n}^{n} b_{0}+\xi_{n}^{n} c_{0}$}

Next, for fixed $n, k \in N$, for all $1 \leq q \leq k$ we take the following presentations:

$$
\begin{aligned}
& \varphi_{k}^{n}=\alpha_{q}^{n, k} \varphi_{k-q}^{n}+\beta_{q}^{n, k} \psi_{k-q}^{n}+\gamma_{q}^{n, k} \xi_{k-q}^{n}+\sum_{m=0}^{q-1} \sum_{i=q-m}^{k-m-1} i_{q}^{n, k}(i, m) a_{i} \\
& \psi_{k}^{n}=\bar{\alpha}_{q}^{n, k} \varphi_{k-q}^{n}+\bar{\beta}_{q}^{n, k} \psi_{k-q}^{n}+\bar{\gamma}_{q}^{n, k} \xi_{k-q}^{n}+\sum_{m=0}^{q-1} \sum_{i=q-m}^{k-m-1} \bar{l}_{q}^{n, k}(i, m) a_{i} \\
& \xi_{k}^{n}=\overline{\bar{\alpha}}_{q}^{n, k} \varphi_{k-q}^{n}+\overline{\bar{\beta}}_{q}^{n, k} \psi_{k-q}^{n}+\overline{\bar{\gamma}}_{q}^{n, k} \xi_{k-q}^{n}+\sum_{m=0}^{q-1} \sum_{i=q-m}^{k-m-1} \overline{\bar{l}}_{q}^{n, k}(i, m) a_{i} .
\end{aligned}
$$

For the presentation (2.3) we obtain the system of difference equations (2.6). 


$$
\begin{aligned}
& \alpha_{q}^{n, k}=-\sigma \alpha_{q-1}^{n, k}+\sigma \beta_{q-1}^{n, k} \\
& \beta_{q}^{n, k}=r \alpha_{q-1}^{n, k}-\beta_{q-1}^{n, k}-a_{0} \gamma_{q-1}^{n, k}-\sum_{i=1}^{q-1} \gamma_{q-1-i}^{n, k}\left(\begin{array}{c}
n-k+q-1 \\
i
\end{array}\right) a_{i} \\
& \gamma_{q}^{n, k}=a_{0} \beta_{q-1}^{n, k}-b \gamma_{q-1}^{n, k}+\sum_{i=1}^{q-1} \beta_{q-1-i}^{n, k}\left(\begin{array}{c}
n-k+q-1 \\
i
\end{array}\right) a_{i} \\
& l_{q}^{n, k}(i, m)=\left(\begin{array}{c}
n-k+i+m \\
i
\end{array}\right)\left[\beta_{m}^{n, k} \xi_{k-m-i-1}^{n}-\gamma_{m}^{n, k} \psi_{k-m-i-1}^{n}\right]
\end{aligned}
$$

with the initial values $\alpha_{0}^{n, k}=1, \beta_{0}^{n, k}=0, \gamma_{0}^{n, k}=0, \imath_{0}^{n, k}=0$.

For the presentation (2.4) we obtain the system of difference equations:

$$
\begin{aligned}
& \bar{\alpha}_{q}^{n, k}=-\sigma \bar{\alpha}_{q-1}^{n, k}+\sigma \bar{\beta}_{q-1}^{n, k} \\
& \bar{\beta}_{q}^{n, k}=r \bar{\alpha}_{q-1}^{n, k}-\bar{\beta}_{q-1}^{n, k}-a_{0} \bar{\gamma}_{q-1}^{n, k}-\sum_{i=1}^{q-1} \bar{\gamma}_{q-1-i}^{n, k}\left(\begin{array}{c}
n-k+q-1 \\
i
\end{array}\right) a_{i} \\
& \bar{\gamma}_{q}^{n, k}=a_{0} \bar{\beta}_{q-1}^{n, k}-b \bar{\gamma}_{q-1}^{n, k}+\sum_{i=1}^{q-1} \bar{\beta}_{q-1-i}^{n, k}\left(\begin{array}{c}
n-k+q-1 \\
i
\end{array}\right) a_{i} \\
& \bar{t}_{q}^{n, k}(i, m)=\left(\begin{array}{c}
n-k+i+m \\
i
\end{array}\right)\left[\bar{\beta}_{m}^{n, k} \xi_{k-m-i-1}^{n}-\bar{\gamma}_{m}^{n, k} \psi_{k-m-i-1}^{n}\right]
\end{aligned}
$$

with the initial values $\bar{\alpha}_{0}^{n, k}=0, \bar{\beta}_{0}^{n, k}=1, \bar{\gamma}_{0}^{n, k}=0, \bar{\imath}_{0}^{n, k}=0$.

For the presentation (2.5) we obtain the system of difference equations:

$$
\begin{aligned}
& \overline{\bar{\alpha}}_{q}^{n, k}=-\sigma \overline{\bar{\alpha}}_{q-1}^{n, k}+\sigma \overline{\bar{\beta}}_{q-1}^{n, k} \\
& \overline{\bar{\beta}}_{q}{ }^{n, k}=r \overline{\bar{\alpha}}_{q-1}^{n, k}-\overline{\bar{\beta}}_{q-1}^{n, k}-a_{0} \overline{\bar{\gamma}}_{q-1}^{n, k}-\sum_{i=1}^{q-1} \overline{\bar{\gamma}}_{q-1-i}^{n, k}\left(\begin{array}{c}
n-k+q-1 \\
i
\end{array}\right) a_{i} \\
& \overline{\bar{\gamma}}_{q}^{n, k}=a_{0} \overline{\bar{\beta}}_{q-1}^{n, k}-b \overline{\bar{\gamma}}_{q-1}^{n, k}+\sum_{i=1}^{q-1} \overline{\bar{\beta}}_{q-1-i}^{n, k}\left(\begin{array}{c}
n-k+q-1 \\
i
\end{array}\right) a_{i} \\
& \overline{\bar{l}}_{q}^{n, k}(i, m)=\left(\begin{array}{c}
n-k+i+m \\
i
\end{array}\right)\left[\overline{\bar{\beta}}_{m}^{n, k} \xi_{k-m-i-1}^{n}-\overline{\bar{\gamma}}_{m}^{n, k} \psi_{k-m-i-1}^{n}\right]
\end{aligned}
$$

with the initial values $\overline{\bar{\alpha}}_{0}^{n, k}=0, \overline{\bar{\beta}}_{0}^{n, k}=0, \overline{\bar{\gamma}}_{0}^{n, k}=1, \overline{\bar{\imath}}_{0}^{n, k}=0$. 
For $k=q$, directly from the presentations (2.3), (2.4) and (2.5), the inequality $k-m-1<k-m$, together with $\varphi_{0}^{n}=1, \psi_{0}^{n}=0, \xi_{0}^{n}=0$, implies:

2.2. $\varphi_{k}^{n}=\alpha_{k}^{n, k}, \psi_{k}^{n}=\bar{\alpha}_{k}^{n, k}$ and $\xi_{k}^{n}=\overline{\bar{\alpha}}_{k}^{n, k}$.

For $n=k=q$, 2.1. and 2.2. imply:

2.3. $a_{n}=\alpha_{n}^{n, n} a_{0}+\bar{\alpha}_{n}^{n, n} b_{0}+\overline{\bar{\alpha}}_{n}^{n, n} c_{0}$.

With all this, the question of finding a solution for $a_{n}$ is transformed to the question of solving the systems of difference equations (2.6), (2.7) and (2.8).

We start with the system (2.6). By finding expressions for $\alpha_{q}^{n, k}, \beta_{q}^{n, k}$ and $\gamma_{q}^{n, k}$ for several small values of $n, k, q$ we found that there are some regularities, and after long calculations, we obtained the following presentations:

where:

$$
\alpha_{q}^{n, k}=X_{q}^{n, k}+L_{q}^{n, k} \quad \beta_{q}^{n, k}=Y_{q}^{n, k}+M_{q}^{n, k} \quad \gamma_{q}^{n, k}=N_{q}^{n, k},
$$

$$
\begin{aligned}
& X_{q}^{n, k}=(-1)^{q-1} \sigma\left\{-(\sigma+r)^{q-1}+\sum_{m=1}^{\left[\frac{q-1}{2}\right]} \sum_{j=m+1}^{q-m}\left(\begin{array}{c}
q-j \\
m
\end{array}\right)\left(\begin{array}{c}
j-1 \\
m-1
\end{array}\right) \sigma^{q-j-1}\left(r^{j}-r^{m}\right)\right\} \\
& Y_{q}^{n, k}=(-1)^{q-1}\left\{(\sigma+r)^{q-1} r-\sum_{m=1}^{\left[\frac{q}{2}\right]_{j=m+1}} \sum_{q-m+1}\left(\begin{array}{c}
q-j \\
m-1
\end{array}\right)\left(\begin{array}{c}
j-1 \\
m-1
\end{array}\right) \sigma^{q-j}\left(r^{j}-r^{m}\right)\right\}
\end{aligned}
$$

and $L_{q}^{n, k}, M_{q}^{n, k}, N_{q}^{n, k}$ satisfy the following system of difference equations: $L_{q}^{n, k}=-\sigma L_{q-1}^{n, k}+\sigma M_{q-1}^{n, k}$

$$
\begin{aligned}
& M_{q}^{n, k}=r L_{q-1}^{n, k}-M_{q-1}^{n, k}-a_{0} N_{q-1}^{n, k}-\sum_{i=1}^{q-1} N_{q-1-i}^{n, k}\left(\begin{array}{c}
n-k+q-1 \\
i
\end{array}\right) a_{i} \\
& N_{q}^{n, k}=a_{0} Y_{q-1}^{n, k}+a_{0} M_{q-1}^{n, k}-b N_{q-1}^{n, k}+\sum_{i=1}^{q-1}\left(Y_{q-1-i}^{n, k}+M_{q-1-i}^{n, k}\right)\left(\begin{array}{c}
n-k+q-1 \\
i
\end{array}\right) a_{i}
\end{aligned}
$$

with $X_{0}^{n, k}=1, Y_{0}^{n, k}=0, L_{0}^{n, k}=0, M_{0}^{n, k}=0, N_{0}^{n, k}=0$.

By 2.2. and 2.3., it is enough to find $\varphi_{n}^{n}=\alpha_{n}^{n, n}=X_{n}^{n, n}+L_{n}^{n, n}$, and since $X_{n}^{n, n}$ is known, we have to find $L_{n}^{n, n}$. Each $L_{q}^{n, k}, M_{q}^{n, k}, N_{q}^{n, k}$ is a polynomial with 
variables $\sigma, r, b, a_{0}, b_{0}$ and $c_{0}$. For convenience with the signs, we use the notation: $\hat{L}_{q}^{n, k}=(-1)^{q-1} L_{q}^{n, k}$. By suitably grouping the parts in these polynomials and calculating the first several of them, we found that:

$$
\begin{aligned}
\hat{L}_{1}^{n, n} & =\hat{L}_{2}^{n, n}=\hat{L}_{3}^{n, n}=0 ; \quad \hat{L}_{4}^{n, n}=\sigma\left\{r a_{0}^{2}\right\} \\
\hat{L}_{5}^{n, n} & =\sigma\left\{2\left(r a_{0}^{2}\right)+6 \sigma\left(r a_{0}^{2}\right)+b\left(r a_{0}^{2}\right)-5 \sigma r a_{0} b_{0}\right\} ; \\
\hat{L}_{6}^{n, n} & =\sigma\left\{3\left(r a_{0}^{2}\right)+16 \sigma\left(r a_{0}^{2}\right)+2 b\left(r a_{0}^{2}\right)+32 \sigma^{2}\left(r a_{0}^{2}\right)+8 \sigma b\left(r a_{0}^{2}\right)+b^{2}\left(r a_{0}^{2}\right)\right. \\
& +11 \sigma r\left(r a_{0}^{2}\right)-\left(r a_{0}^{2}\right) a_{0}^{2}-9 \sigma c_{0}\left(r a_{0}^{2}\right)-21 \sigma r a_{0} b_{0}-37 \sigma\left(\sigma r a_{0} b_{0}\right) \\
& \left.-6 b\left(\sigma r a_{0} b_{0}\right)+8 \sigma^{2} r b_{0}^{2}\right\} ; \\
\hat{L}_{7}^{n, n} & =\sigma\left\{4\left(r a_{0}^{2}\right)+27 \sigma\left(r a_{0}^{2}\right)+3 b\left(r a_{0}^{2}\right)+82 \sigma^{2}\left(r a_{0}^{2}\right)+18 \sigma b\left(r a_{0}^{2}\right)+2 b^{2}\left(r a_{0}^{2}\right)\right. \\
& +122 \sigma^{3}\left(r a_{0}^{2}\right)+40 \sigma^{2} b\left(r a_{0}^{2}\right)+9 \sigma b^{2}\left(r a_{0}^{2}\right)+b^{3}\left(r a_{0}^{2}\right)+45 \sigma r\left(r a_{0}^{2}\right) \\
& +108 \sigma \sigma r\left(r a_{0}^{2}\right)+15 b \sigma r\left(r a_{0}^{2}\right)-3\left(r a_{0}^{2}\right) a_{0}^{2}-16 \sigma\left(r a_{0}^{2}\right) a_{0}^{2}-2 b\left(r a_{0}^{2}\right) a_{0}^{2} \\
& -39 \sigma c_{0}\left(r a_{0}^{2}\right)-74 \sigma \sigma c_{0}\left(r a_{0}^{2}\right)-27 b \sigma c_{0}\left(r a_{0}^{2}\right)+28 \sigma a_{0} b_{0}\left(r a_{0}^{2}\right)-60 \sigma r a_{0} b_{0} \\
& -173 \sigma\left(\sigma r a_{0} b_{0}\right)-27 b\left(\sigma r a_{0} b_{0}\right)-161 \sigma^{2}\left(\sigma r a_{0} b_{0}\right)-47 \sigma b\left(\sigma r a_{0} b_{0}\right) \\
& -7 b^{2}\left(\sigma r a_{0} b_{0}\right)-63 \sigma r\left(\sigma r a_{0} b_{0}\right)+49 \sigma c_{0}\left(\sigma r a_{0} b_{0}\right)+58\left(\sigma^{2} r b_{0}^{2}\right) \\
& \left.+58 \sigma\left(\sigma^{2} r b_{0}^{2}\right)+10 b\left(\sigma^{2} r b_{0}^{2}\right)\right\} ;
\end{aligned}
$$

and for $q>7$, the polynomial $\hat{L}_{q}^{n, n}$ has the form of the polynomial

$$
A \hat{L}_{q-1}^{n, n}+B \hat{L}_{q-2}^{n, n}+C \hat{L}_{q-3}^{n, n}+D \hat{L}_{q-4}^{n, n}
$$

where $A=1+\sigma+b, B=\sigma r-a_{0}^{2}-\sigma c_{0}, C=\sigma a_{0} b_{0}, D=-\sigma^{2} b_{0}^{2}$. So, for $q>7$ we choose to approximate $\hat{L}_{q}^{n, n}$ with the solutions $\hat{L}_{q}^{n, n}(\approx)$ of the difference equation:

$$
\hat{L}_{q}^{n, n}(\approx)=A \hat{L}_{q-1}^{n, n}(\approx)+B \hat{L}_{q-2}^{n, n}(\approx)+C \hat{L}_{q-3}^{n, n}(\approx)+D \hat{L}_{q-4}^{n, n}(\approx)
$$

with the initial values $\hat{L}_{4}^{n, n}, \hat{L}_{5}^{n, n}, \hat{L}_{6}^{n, n} \hat{L}_{7}^{n, n}$.

To solve this difference equation, we take the following representation

$$
\hat{L}_{q}^{n, n}(\approx)=P_{w}^{1} \hat{L}_{q-w}^{n, n}(\approx)+P_{w}^{2} \hat{L}_{q-w-1}^{n, n}(\approx)+P_{w}^{3} \hat{L}_{q-w-2}^{n, n}(\approx)+P_{w}^{4} \hat{L}_{q-w-3}^{n, n}(\approx)
$$

where $1 \leq w \leq q$ and $P_{0}^{1}=1, P_{0}^{2}=0, P_{0}^{3}=0, P_{0}^{4}=0, P_{1}^{1}=A, P_{1}^{2}=B, P_{1}^{3}=C, P_{1}^{4}=D$.

Using the presentation (2.14) we obtain the following system of difference equations: 


$$
\begin{array}{ll}
P_{w}^{1}=A P_{w-1}^{1}+P_{w-1}^{2} & P_{w}^{2}=B P_{w-1}^{1}+P_{w-1}^{3} \\
P_{w}^{3}=C P_{w-1}^{1}+P_{w-1}^{4} & P_{w}^{4}=D P_{w-1}^{1}
\end{array} .
$$

The solutions of the system (2.15) are the polynomials:

$$
\begin{aligned}
& P_{w}^{1}=A^{w}+\sum_{j=1}^{\left[\frac{w}{2}\right.}\left(\begin{array}{c}
w-j \\
j
\end{array}\right) A^{w-2 j} B^{j}+\sum_{j=1}^{\left[\frac{w}{3}\right.}\left(\begin{array}{c}
w-2 j \\
j
\end{array}\right) A^{w-3 j} C^{j}+\sum_{j=1}^{\left[\frac{w}{4}\right.}\left(\begin{array}{c}
w-3 j \\
j
\end{array}\right) A^{w-4 j} D^{j} \\
& \left.+\sum_{m=1}^{\left[\frac{w-2}{3}\right]} \sum_{j=m+1}^{\frac{w-m}{2}}\right]\left(\begin{array}{c}
j \\
m
\end{array}\right)\left(\begin{array}{c}
w-j-m \\
j
\end{array}\right) A^{w-2 j-m} B^{j-m} C^{m} \\
& +\left[\sum_{m=1}^{\left[\frac{w-2}{4}\right]} \sum_{j=m+1}^{\frac{w-2 m}{2}}\right]\left(\begin{array}{c}
j \\
m
\end{array}\right)\left(\begin{array}{c}
w-j-2 m \\
j
\end{array}\right) A^{w-2 j-2 m} B^{j-m} D^{m}
\end{aligned}
$$

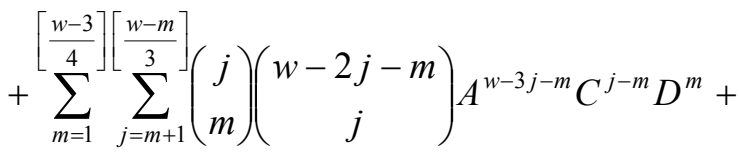

$$
\begin{aligned}
& +\left[\begin{array}{l}
\left.\frac{w-5}{4}\right] \\
s=1
\end{array} \sum_{m=s+1}^{\frac{w-s-2}{3}}\right]\left[\frac{\frac{w-m-s-2}{2}}{\sum_{j=m}^{2}}\left(\begin{array}{c}
m \\
s
\end{array}\right)\left(\begin{array}{c}
j+1 \\
m
\end{array}\right)\left(\begin{array}{c}
w-j-m-s-1 \\
j+1
\end{array}\right) A^{w-2 j-m-s-2} B^{j-m+1} C^{m-s} D^{s}\right. \\
& \left.P_{w}^{2}=\sum_{j=1}^{\left[\frac{w+1}{2}\right.}\right]\left(\begin{array}{c}
w-j \\
j-1
\end{array}\right) A^{w-2 j+1} B^{j}+\sum_{j=1}^{\left[\frac{w+1}{3}\right.}\left(\begin{array}{c}
w-2 j \\
j-1
\end{array}\right) A^{w-3 j+1} C^{j}+\sum_{j=1}^{\left[\frac{w+1}{4}\right]}\left(\begin{array}{c}
w-3 j \\
j-1
\end{array}\right) A^{w-4 j+1} D^{j}
\end{aligned}
$$

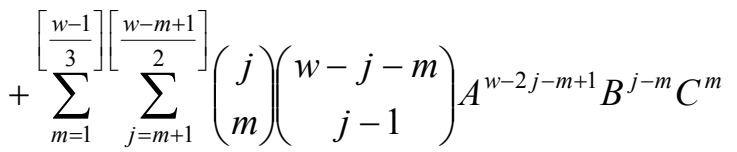

$$
\begin{aligned}
& +\sum_{m=1}^{\left[\frac{w-1}{4}\right]} \sum_{j=m+1}^{\left.\frac{w-2 m+1}{2}\right]}\left(\begin{array}{c}
j \\
m
\end{array}\right)\left(\begin{array}{c}
w-j-2 m \\
j-1
\end{array}\right) A^{w-2 j-2 m+1} B^{j-m} D^{m} \\
& \left.+\sum_{m=1}^{\left[\frac{w-2}{4}\right.} \sum_{j=m+1}^{\frac{w-m+1}{3}}\right]\left(\begin{array}{c}
j \\
m
\end{array}\right)\left(\begin{array}{c}
w-2 j-m \\
j-1
\end{array}\right) A^{w-3 j-m+1} C^{j-m} D^{m} \\
& +\left[\begin{array}{l}
\left.\frac{w-4}{4}\right] \\
s=1
\end{array} \sum_{m=s+1}^{\frac{w-s-1}{3}}\right]\left[\frac{w-m-s-1}{2}\right]\left(\begin{array}{c}
m \\
s
\end{array}\right)\left(\begin{array}{c}
j+1 \\
m
\end{array}\right)\left(\begin{array}{c}
w-j-m-s-1 \\
j
\end{array}\right) A^{w-2 j-m-s-1} B^{j-m+1} C^{m-s} D^{s}
\end{aligned}
$$

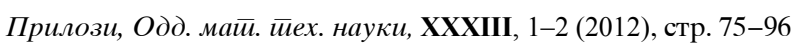




$$
\begin{aligned}
P_{w}^{3}= & \sum_{j=1}^{\left[\frac{w+2}{3}\right]}\left(\begin{array}{c}
w-2 j+1 \\
j-1
\end{array}\right) A^{w-3 j+2} C^{j}+\sum_{j=1}^{\left.\frac{w+2}{4}\right]}\left(\begin{array}{c}
w-3 j+1 \\
j-1
\end{array}\right) A^{w-4 j+2} D^{j} \\
& +\left[\sum_{m=1}^{\frac{w}{3}}\left[\frac{w-m}{2}\right]\left(\begin{array}{c}
j \\
m-1
\end{array}\right)\left(\begin{array}{c}
w-j-m \\
j
\end{array}\right) A^{w-2 j-m} B^{j-m+1} C^{m}\right. \\
& +\sum_{m=1}^{\frac{w}{4}}\left[\frac{w-2 m}{j=m}\right]\left(\begin{array}{c}
j \\
m-1
\end{array}\right)\left(\begin{array}{c}
w-j-2 m \\
j
\end{array}\right) A^{w-2 j-2 m} B^{j-m+1} D^{m} \\
& +\left[\frac{w-1}{4}\right]\left[\frac{w-m-1}{3}\right]\left(\begin{array}{c}
j+1 \\
\sum_{m=1}^{2} \\
j=m
\end{array}\right)\left(\begin{array}{c}
w-2 j-m-1 \\
j
\end{array}\right) A^{w-3 j-m-1} C^{j-m+1} D^{m} \\
& +\left[\frac{w-3}{4}\right]\left[\frac{w-s}{3}\right]\left[\frac{w-m-s}{2}\right]\left(\begin{array}{c}
m \\
j \\
s=1
\end{array}\right)\left(\begin{array}{c}
w-j-m-s \\
m-1
\end{array}\right) A_{m=s+1} \sum_{j=m}^{w-2 j-m-s} B^{j-m+1} C^{m-s} D^{s}
\end{aligned}
$$

and

$$
\begin{aligned}
P_{w}^{4}= & \sum_{j=1}^{\left.\frac{w+3}{4}\right]}\left(\begin{array}{c}
w-3 j+2 \\
j-1
\end{array}\right) A^{w-4 j+3} D^{j} \\
& \left.+\sum_{m=1}^{\frac{w+1}{4}}\right]\left[\frac{w-2 m+1}{2}\right]\left(\begin{array}{c}
j \\
m-1
\end{array}\right)\left(\begin{array}{c}
w-j-2 m+1 \\
j
\end{array}\right) A^{w-2 j-2 m+1} B^{j-m+1} D^{m} \\
& +\sum_{m=1}^{\frac{w}{4}}\left[\frac{w-m}{3}\right] \\
\sum_{j=m}^{3} & {\left[\begin{array}{c}
j \\
m-1
\end{array}\right)\left(\begin{array}{c}
w-2 j-m \\
j
\end{array}\right) A^{w-3 j-m} C^{j-m+1} D^{m} . } \\
& \left.+\sum_{s=1}^{\frac{w-2}{4}}\right]\left[\frac{w-s+1}{\sum_{m=s+1}^{3}}\right]\left[\frac{w-m-s+1}{\sum_{j=m}^{2}}\right]\left(\begin{array}{c}
m-1 \\
s-1
\end{array}\right)\left(\begin{array}{c}
j \\
m-1
\end{array}\right)\left(\begin{array}{c}
w-j-m-s+1 \\
j
\end{array}\right) A^{w-2 j-m-s+1} B^{j-m+1} C^{m-s} D^{s} .
\end{aligned}
$$

For $w=q-7$ :

$$
\hat{L}_{q}^{n, n}(\approx)=P_{q-7}^{1} \hat{L}_{7}^{n, n}+P_{q-7}^{2} \hat{L}_{6}^{n, n}+P_{q-7}^{3} \hat{L}_{5}^{n, n}+P_{q-7}^{4} \hat{L}_{4}^{n, n},
$$

and from 2.2., for $n=q$, we obtain: 


$$
\begin{aligned}
\varphi_{n}^{n}(\approx)= & \alpha_{n}^{n, n}(\approx)=X_{n}^{n, n}+L_{n}^{n, n}(\approx) \\
& =(-1)^{n-1} \sigma\left\{-(\sigma+r)^{n-1}+\sum_{m=1}^{\left[\frac{n-1}{2}\right.} \sum_{j=m+1}^{n-m}\left(\begin{array}{c}
n-j \\
m
\end{array}\right)\left(\begin{array}{c}
j-1 \\
m-1
\end{array}\right) \sigma^{n-j-1}\left(r^{j}-r^{m}\right)\right\} \\
& +(-1)^{n-1}\left\{P_{n-7}^{1} \hat{L}_{7}^{n, n}+P_{n-7}^{2} \hat{L}_{6}^{n, n}+P_{n-7}^{3} \hat{L}_{5}^{n, n}+P_{n-7}^{4} \hat{L}_{4}^{n, n}\right\} .
\end{aligned}
$$

Next, we continue with the system (2.7). Similarly as above we set:

where:

$$
\bar{\alpha}_{q}^{n, k}=\bar{X}_{q}^{n, k}+\bar{L}_{q}^{n, k}, \quad \bar{\beta}_{q}^{n, k}=\bar{Y}_{q}^{n, k}+\bar{M}_{q}^{n, k}, \quad \bar{\gamma}_{q}^{n, k}=\bar{N}_{q}^{n, k}
$$

$$
\begin{aligned}
& \bar{X}_{q}^{n, k}=(-1)^{q-1} \sigma\left\{(\sigma+r)^{q-1}-\sum_{m=1}^{\left.\frac{q}{2}\right]} \sum_{j=m}^{q-m}\left(\begin{array}{c}
q-1-j \\
m-1
\end{array}\right)\left(\begin{array}{c}
j \\
m-1
\end{array}\right) \sigma^{q-j-1}\left(r^{j}-r^{m-1}\right)\right\} \\
& \bar{Y}_{q}^{n, k}=(-1)^{q-1}\left\{-(\sigma+r)^{q-1} r+\left(r^{q}-1\right)+\sum_{m=2}^{\left[\frac{q+1}{2}\right.} \sum_{j=m}^{q-m+1}\left(\begin{array}{c}
q-1-j \\
m-2
\end{array}\right)\left(\begin{array}{c}
j \\
m-1
\end{array}\right) \sigma^{q-j}\left(r^{j}-r^{m-1}\right)\right\}
\end{aligned}
$$

and $\bar{L}_{q}^{n, k}, \bar{M}_{q}^{n, k}, \bar{N}_{q}^{n, k}$ satisfy the difference equations (2.16),

$$
\begin{aligned}
& \bar{L}_{q}^{n, k}=-\sigma \bar{L}_{q-1}^{n, k}+\sigma \bar{M}_{q-1}^{n, k} \\
& \bar{M}_{q}^{n, k}=r \bar{L}_{q-1}^{n, k}-\bar{M}_{q-1}^{n, k}-a_{0} \bar{N}_{q-1}^{n, k}-\sum_{i=1}^{q-1} \bar{N}_{q-1-i}^{n, k}\left(\begin{array}{c}
n-k+q-1 \\
i
\end{array}\right) a_{i} \\
& \bar{N}_{q}^{n, k}=a_{0} \bar{Y}_{q-1}^{n, k}+a_{0} \bar{M}_{q-1}^{n, k}-b \bar{N}_{q-1}^{n, k}+\sum_{i=1}^{q-1}\left(\bar{Y}_{q-1-i}^{n, k}+\bar{M}_{q-1-i}^{n, k}\right)\left(\begin{array}{c}
n-k+q-1 \\
i
\end{array}\right) a_{i}
\end{aligned}
$$

with $\bar{X}_{0}^{n, k}=0, \bar{Y}_{0}^{n, k}=1, \bar{L}_{0}^{n, k}=0, \bar{M}_{0}^{n, k}=0, \bar{N}_{0}^{n, k}=0$. The system (2.16) is the system (2.12), but with different notations, and different initial values.

By 2.2. and 2.3., it is enough to find $\bar{\varphi}_{n}^{n}=\bar{\alpha}_{n}^{n, n}=\bar{X}_{n}^{n, n}+\bar{L}_{n}^{n, n}$, and since $\bar{X}_{n}^{n, n}$ is known, we have to find $\bar{L}_{n}^{n, n}$. Using the notation: $\hat{\bar{L}}_{q}^{n, k}=(-1)^{q-1} \bar{L}_{q}^{n, k}$, we calculated $\hat{\bar{L}}_{q}^{n, k}$ for, $q$ from 1 to 6 :

$\hat{\bar{L}}_{1}^{n, n}=\hat{\bar{L}}_{2}^{n, n}=0 ; \quad \hat{\bar{L}}_{3}^{n, n}=\sigma\left(-a_{0}^{2}\right) ; \quad \hat{\bar{L}}_{4}^{n, n}=\sigma\left\{-2\left(a_{0}^{2}\right)-4 \sigma\left(a_{0}^{2}\right)-b\left(a_{0}^{2}\right)+3 \sigma a_{0} b_{0}\right\} ;$ 


$$
\begin{aligned}
{\hat{\overline{L_{5}}}}^{n, n} & =\sigma\left\{-3\left(a_{0}^{2}\right)-10 \sigma\left(a_{0}^{2}\right)-2 b\left(a_{0}^{2}\right)-11 \sigma^{2}\left(a_{0}^{2}\right)-5 \sigma b\left(a_{0}^{2}\right)-b^{2}\left(a_{0}^{2}\right)-6 \sigma r\left(a_{0}^{2}\right)\right. \\
& \left.+\left(a_{0}^{2}\right) a_{0}^{2}+4 \sigma c_{0}\left(a_{0}^{2}\right)+12 \sigma a_{0} b_{0}+13 \sigma\left(\sigma a_{0} b_{0}\right)+4 b\left(\sigma a_{0} b_{0}\right)-3 \sigma^{2} b_{0}^{2}\right\} ; \\
\hat{\bar{L}}_{6}^{n, n} & =\sigma\left\{-4\left(a_{0}^{2}\right)-18 \sigma\left(a_{0}^{2}\right)-3 b\left(a_{0}^{2}\right)-34 \sigma^{2}\left(a_{0}^{2}\right)-12 \sigma b\left(a_{0}^{2}\right)-2 b^{2}\left(a_{0}^{2}\right)-26 \sigma^{3}\left(a_{0}^{2}\right)\right. \\
& -16 \sigma^{2} b\left(a_{0}^{2}\right)-6 \sigma b^{2}\left(a_{0}^{2}\right)-b^{3}\left(a_{0}^{2}\right)-24 \sigma r\left(a_{0}^{2}\right)-38 \sigma \sigma r\left(a_{0}^{2}\right)-9 b \sigma r\left(a_{0}^{2}\right) \\
& +3\left(a_{0}^{2}\right) a_{0}^{2}+11 \sigma\left(a_{0}^{2}\right) a_{0}^{2}+2 b\left(a_{0}^{2}\right) a_{0}^{2}+18 \sigma c_{0}\left(a_{0}^{2}\right)+24 \sigma \sigma c_{0}\left(a_{0}^{2}\right)+12 b \sigma c_{0}\left(a_{0}^{2}\right) \\
& -15 \sigma a_{0} b_{0}\left(a_{0}^{2}\right)+33 \sigma a_{0} b_{0}+62 \sigma\left(\sigma a_{0} b_{0}\right)+17 b\left(\sigma a_{0} b_{0}\right)+38 \sigma^{2}\left(\sigma a_{0} b_{0}\right) \\
& +19 \sigma b\left(\sigma a_{0} b_{0}\right)+5 b^{2}\left(\sigma a_{0} b_{0}\right)+25 \sigma r\left(\sigma a_{0} b_{0}\right)-15 \sigma c_{0}\left(\sigma a_{0} b_{0}\right)-21\left(\sigma^{2} b_{0}^{2}\right) \\
& \left.-13 \sigma\left(\sigma^{2} b_{0}^{2}\right)-4 b\left(\sigma^{2} b_{0}^{2}\right)\right\} .
\end{aligned}
$$

By the same argument as above, we arrive to the same difference equations as (2.13) and (2.14), but for $q>6$, and to the same equation (2.15). With all this we obtain:

$$
\begin{aligned}
& \psi_{n}^{n}(\approx)=\bar{\alpha}_{n}^{n, n}(\approx)=\bar{X}_{n}^{n, n}+\bar{L}_{n}^{n, n}(\approx) \\
& =(-1)^{n-1} \sigma\left\{(\sigma+r)^{n-1}-\sum_{m=1}^{\left[\frac{n}{2}\right]_{j=m}^{n-m}}\left(\begin{array}{c}
n-1-j \\
m-1
\end{array}\right)\left(\begin{array}{c}
j \\
m-1
\end{array}\right) \sigma^{n-j-1}\left(r^{j}-r^{m-1}\right)\right\} \\
& +(-1)^{n-1}\left\{P_{n-6}^{1} \hat{\bar{L}}_{6}^{n, n}+P_{n-6}^{2} \hat{\bar{L}}_{5}^{n, n}+P_{n-6}^{3} \hat{\bar{L}}_{4}^{n, n}+P_{n-6}^{4} \hat{\bar{L}}_{3}^{n, n}\right\} .
\end{aligned}
$$

For the system (2.8), we set:

$$
\overline{\bar{\alpha}}_{q}^{n, k}=\overline{\bar{L}}_{q}^{n, k}, \quad \overline{\bar{\beta}}_{q}^{n, k}=\overline{\bar{M}}_{q}^{n, k}, \quad \overline{\bar{\gamma}}_{q}^{n, k}=(-b)^{q}+\overline{\bar{N}}_{q}^{n, k}
$$

where $\overline{\bar{L}}_{q}^{n, k}, \overline{\bar{M}}_{q}^{n, k}, \overline{\bar{N}}_{q}^{n, k}$ satisfy the difference equations (2.17),

$$
\begin{aligned}
& \overline{\bar{L}}_{q}^{n, k}=-\sigma \overline{\bar{L}}_{q-1}^{n, k}+\sigma \overline{\bar{M}}_{q-1}^{n, k} \\
& \overline{\bar{M}}_{q}^{n, k}=r \overline{\bar{L}}_{q-1}^{n, k}-\overline{\bar{M}}_{q-1}^{n, k}-a_{0} \overline{\bar{N}}_{q-1}^{n, k}-\sum_{i=1}^{q-1} \overline{\bar{N}}_{q-1-i}^{n, k}\left(\begin{array}{c}
n-k+q-1 \\
i
\end{array}\right) a_{i} \\
& \overline{\bar{N}}_{q}^{n, k}=a_{0} \overline{\bar{M}}_{q-1}^{n, k}-b \overline{\bar{N}}_{q-1}^{n, k}+\sum_{i=1}^{q-1} \overline{\bar{M}}_{q-1-i}^{n, k}\left(\begin{array}{c}
n-k+q-1 \\
i
\end{array}\right) a_{i}
\end{aligned}
$$

with $\overline{\bar{L}}_{0}^{n, k}=0, \overline{\bar{M}}_{0}^{n, k}=0, \overline{\bar{N}}_{0}^{n, k}=0$. The system (2.17) is analogous to the system (2.12) (i.e. the system (2.16)), but with different notations, and different initial values. 
By 2.2. and 2.3., it is enough to find $\overline{\bar{\varphi}}_{n}^{n}=\overline{\bar{\alpha}}_{n}^{n, n}=\overline{\bar{L}}_{n}^{n, n}$. Using the notation: $\hat{\overline{\bar{L}}}_{q}^{n, k}=(-1)^{q-1} \overline{\bar{L}}_{q}^{n, k}$, we calculated $\hat{\bar{L}}_{q}^{n, k}$ for $q$ from 1 to 5:

$$
\begin{aligned}
\hat{\overline{\bar{L}}}_{1}^{n, n} & =0 ; \quad \hat{\overline{\bar{L}}}_{2}^{n, n}=\sigma a_{0} ; \quad \hat{\overline{\bar{L}}}_{3}^{n, n}=\sigma\left\{\left(a_{0}\right)+2 \sigma\left(a_{0}\right)+b\left(a_{0}\right)-\sigma b_{0}\right\} ; \\
\hat{\overline{\bar{L}}}_{4}^{n, n} & =\sigma\left\{\left(a_{0}\right)+2 \sigma\left(a_{0}\right)+b\left(a_{0}\right)+3 \sigma^{2}\left(a_{0}\right)+3 \sigma b\left(a_{0}\right)+b^{2}\left(a_{0}\right)+2 \sigma r\left(a_{0}\right)\right. \\
& \left.-\left(a_{0}\right) a_{0}^{2}-\sigma c_{0}\left(a_{0}\right)-2 \sigma b_{0}-2 \sigma\left(\sigma b_{0}\right)-2 b\left(\sigma b_{0}\right)\right\} ; \\
\hat{\overline{\bar{L}}}_{5}^{n, n} & =\sigma\left\{\left(a_{0}\right)+2 \sigma\left(a_{0}\right)+b\left(a_{0}\right)+3 \sigma^{2}\left(a_{0}\right)+3 \sigma b\left(a_{0}\right)+b^{2}\left(a_{0}\right)+4 \sigma^{3}\left(a_{0}\right)\right. \\
& +6 \sigma^{2} b\left(a_{0}\right)+4 \sigma b^{2}\left(a_{0}\right)+b^{3}\left(a_{0}\right)+4 \sigma r\left(a_{0}\right)+6 \sigma \sigma r\left(a_{0}\right)+4 b \sigma r\left(a_{0}\right) \\
& -2\left(a_{0}\right) a_{0}^{2}-7 \sigma\left(a_{0}\right) a_{0}^{2}-2 b\left(a_{0}\right) a_{0}^{2}-2 \sigma c_{0}\left(a_{0}\right)-3 \sigma \sigma c_{0}\left(a_{0}\right)-4 b \sigma c_{0}\left(a_{0}\right) \\
& +7 \sigma a_{0} b_{0}\left(a_{0}\right)-3 \sigma b_{0}-4 \sigma\left(\sigma b_{0}\right)-5 b\left(\sigma b_{0}\right)-3 \sigma^{2}\left(\sigma b_{0}\right)-5 \sigma b\left(\sigma b_{0}\right) \\
& \left.-3 b^{2}\left(\sigma b_{0}\right)-2 \sigma r\left(\sigma b_{0}\right)+\sigma\left(\sigma b_{0}\right) c_{0}\right\} .
\end{aligned}
$$

Again, by the same argument as above, we arrive to the same difference equations as (2.13) and (2.14), but for $q>5$, and to the same equation (2.15). With all this, we have

$$
\xi_{n}^{n}(\approx)=\overline{\bar{\alpha}}_{n}^{n, n}(\approx)=\overline{\bar{L}}_{n}^{n, n}(\approx)=(-1)^{n-1}\left\{P_{n-5}^{1} \hat{\bar{L}}_{5}^{n, k}+P_{n-5}^{2} \hat{\bar{L}}_{4}^{n, n}+P_{n-5}^{3} \hat{\bar{L}}_{3}^{n, n}+P_{n-5}^{4} \hat{\bar{L}}_{2}^{n, n}\right\} \text {. }
$$

The above discussion and 2.3., produce the following approximation

$$
a_{n}(\approx)=\alpha_{n}^{n, n}(\approx) a_{0}+\bar{\alpha}_{n}^{n, n}(\approx) b_{0}+\overline{\bar{\alpha}}_{n}^{n, n}(\approx) c_{0}
$$

for $a_{n}, n>7$.

For $j \in\{1,2,3,4,5,6,7\}$ we calculate from (1.3) the exact values of $a_{j}$ and we set $a_{j}(\approx)=a_{j}$ for $j \in\{0,1,2,3,4,5,6,7\}$.

\section{THE COEFFICIENT $c_{n}$}

For the coefficients $c_{n}$ we consider the system of difference equations (2.1).

For an $n \in N$ and for any $1 \leq k \leq n$ we represent the coefficients $c_{n}$ as: 


$$
\begin{aligned}
c_{n} & =\overline{\bar{\varphi}}_{k}^{n} a_{n-k}+\overline{\bar{\psi}}_{k}^{n} b_{n-k}+\overline{\bar{\xi}}_{k}^{n} c_{n-k}+\sum_{m=0}^{k-1} \sum_{i=k-m}^{n-m-1} \overline{\bar{\tau}}_{k}^{n}(i, m) a_{i} b_{n-i-m-1} \\
& +\sum_{m=0}^{k-1} \sum_{i=k-m}^{n-m-1} \overline{\bar{\pi}}_{k}^{n}(i, m) a_{i} c_{n-i-m-1}
\end{aligned}
$$

where $\overline{\bar{\varphi}}_{k}^{n}, \overline{\bar{\psi}}_{k}^{n}, \overline{\bar{\xi}}_{k}^{n}, \overline{\bar{\tau}}_{k}^{n}(i . m), \overline{\bar{\pi}}_{k}^{n}(i, m)$ are new variables. For the new variables we obtain the new systems of difference equations:

$$
\begin{aligned}
& \overline{\bar{\varphi}}_{k}^{n}=-\sigma \overline{\bar{\varphi}}_{k-1}^{n}+r \overline{\bar{\psi}}_{k-1}^{n} \\
& \overline{\bar{\psi}}_{k}^{n}=\sigma \overline{\bar{\varphi}}_{k-1}^{n}-\overline{\bar{\psi}}_{k-1}^{n}+a_{0} \overline{\bar{\xi}}_{k-1}^{n}+\sum_{i=1}^{k-1} a_{i}\left(\begin{array}{c}
n-k+i \\
i
\end{array}\right) \overline{\bar{\xi}}_{k-1-i}^{n} \\
& \overline{\bar{\xi}}_{k}^{n}=-a_{0} \overline{\bar{\psi}}_{k-1}^{n}-b \overline{\bar{\xi}}_{k-1}^{n}-\sum_{i=1}^{k-1} a_{i}\left(\begin{array}{c}
n-k+i \\
i
\end{array}\right) \overline{\bar{\psi}}_{k-1-i}^{n} \\
& \overline{\bar{\tau}}_{k}^{n}(i, m)=\overline{\bar{\xi}}_{m}^{n}\left(\begin{array}{c}
n-m-1 \\
i
\end{array}\right), \quad \overline{\bar{\pi}}_{k}^{n}(i, m)=-\overline{\bar{\psi}}_{m}^{n}\left(\begin{array}{c}
n-m-1 \\
i
\end{array}\right)
\end{aligned}
$$

with $\overline{\bar{\varphi}}_{0}^{n}=0, \overline{\bar{\psi}}_{0}^{n}=0, \overline{\bar{\xi}}_{0}^{n}=1$, and transform the presentation of $c_{n}$ to:

$$
\begin{aligned}
c_{n} & =\overline{\bar{\varphi}}_{k}^{n} a_{n-k}+\overline{\bar{\psi}}_{k}^{n} b_{n-k}+\overline{\bar{\xi}}_{k}^{n} c_{n-k}+\sum_{m=0}^{k-1} \sum_{i=k-m}^{n-m-1}\left(\begin{array}{c}
n-1-m \\
i
\end{array}\right) \overline{\bar{\xi}}_{m}^{n} a_{i} b_{n-i-m-1} \\
& -\sum_{m=0}^{k-1} \sum_{i=k-t m}^{n-m-1}\left(\begin{array}{c}
n-1-m \\
i
\end{array}\right) \overline{\bar{\psi}}_{m}^{n} a_{i} c_{n-i-m-1} .
\end{aligned}
$$

For $n=k$, the inequality $n-m-1<n-m$, implies:

3.1. $c_{n}=\overline{\bar{\varphi}}_{n}^{n} a_{0}+\overline{\bar{\psi}}_{n}^{n} b_{0}+\overline{\bar{\xi}}_{n}^{n} c_{0}$.

The systems (3.1) are the same as the systems (2.2), with different letters and different initial values. We use the following presentations as for the coefficient $a_{\mathrm{n}}$, with different symbols for all $1 \leq q \leq k$, at fixed $n, k \in N$ :

$$
\begin{aligned}
& \overline{\bar{\varphi}}_{k}^{n}=\rho_{q}^{n, k} \overline{\bar{\varphi}}_{k-q}^{n}+\mu_{q}^{n, k} \overline{\bar{\psi}}_{k-q}^{n}+\delta_{q}^{n, k} \overline{\bar{\xi}}_{k-q}^{n}+\sum_{m=0}^{q-1} \sum_{i=q-m}^{k-m-1} \Delta_{q}^{n, k}(i, m) a_{i} \\
& \overline{\bar{\psi}}_{k}^{n}=\bar{\rho}_{q}^{n, k} \overline{\bar{\varphi}}_{k-q}^{n}+\bar{\mu}_{q}^{n, k} \overline{\bar{\psi}}_{k-q}^{n}+\bar{\delta}_{q}^{n, k} \overline{\bar{\xi}}_{k-q}^{n}+\sum_{m=0}^{q-1} \sum_{i=q-m}^{k-m-1} \bar{\Delta}_{q}^{n, k}(i, m) a_{i} \\
& \overline{\bar{\xi}}_{k}^{n}=\overline{\bar{\rho}}_{q}^{n, k} \overline{\bar{\varphi}}_{k-q}^{n}+\overline{\bar{\mu}}_{q}^{n, k} \overline{\bar{\psi}}_{k-q}^{n}+\overline{\bar{\delta}}_{q}^{n, k} \overline{\bar{\xi}}_{k-q}^{n}+\sum_{m=0}^{q-1} \sum_{i=q-m}^{k-m-1} \overline{\bar{\Delta}}_{q}^{n, k}(i, m) a_{i}
\end{aligned}
$$




$$
\begin{aligned}
& \rho_{q}^{n, k}=U_{q}^{n, k}+S_{q}^{n, k} \\
& \mu_{q}^{n, k}=V_{q}^{n, k}+K_{q}^{n, k} \\
& \delta_{q}^{n, k}=T_{q}^{n, k}
\end{aligned}
$$$$
\bar{\rho}_{q}^{n, k}=\bar{U}_{q}^{n, k}+\bar{S}_{q}^{n, k}
$$$$
\overline{\bar{\rho}}_{q}^{n, k}=\overline{\bar{S}}_{q}^{n, k}
$$$$
\bar{\mu}_{q}^{n, k}=\bar{V}_{q}^{n, k}+\bar{K}_{q}^{n, k}
$$$$
\overline{\bar{\mu}}_{q}^{n, k}=\overline{\bar{K}}_{q}^{n, k}
$$$$
\bar{\delta}_{q}^{n, k}=\bar{T}_{q}^{n, k}
$$$$
\overline{\bar{\delta}}_{q}^{n, k}=(-b)^{q}+\overline{\bar{T}}_{q}^{n, k},
$$

with the initial values:

$$
\begin{aligned}
& \rho_{0}^{n, k}=\bar{\mu}_{0}^{n, k}=\overline{\bar{\delta}}_{0}^{n, k}=1, \bar{\rho}_{0}^{n, k}=\overline{\bar{\rho}}_{0}^{n, k}=\mu_{0}^{n, k}=\overline{\bar{\mu}}_{0}^{n, k}=\delta_{0}^{n, k}=\bar{\delta}_{0}^{n, k}=0, \\
& U_{0}^{n, k}=\bar{V}_{0}^{n, k}=1, \bar{U}_{0}^{n, k}=V_{0}^{n, k}=S_{0}^{n, k}=K_{0}^{n, k}=\bar{S}_{0}^{n, k}=\bar{K}_{0}^{n, k}=\overline{\bar{S}}_{0}^{n, k}=\overline{\bar{K}}_{0}^{n, k}=0, \\
& T_{0}^{n, k}=\bar{T}_{0}^{n, k}=\bar{T}_{0}^{n, k}=\Delta_{0}^{n, k}=\bar{\Delta}_{0}^{n, k}=\overline{\bar{\Delta}}_{0}^{n, k}=0 .
\end{aligned}
$$

For $q=k=n, \overline{\bar{\varphi}}_{n}^{n}=\delta_{n}^{n, n}, \quad \overline{\bar{\psi}}_{n}^{n}=\bar{\delta}_{n}^{n, n}, \overline{\bar{\xi}}_{n}^{n}=\overline{\bar{\delta}}_{n}^{n, n}$, and by analogous discussion as for the coefficient $a_{n}$, we obtain the following approximation for the coefficient $c_{n}, n>5$,

$$
\begin{aligned}
c_{n}(\approx) & =\delta_{n}^{n, n} a_{0}+\bar{\delta}_{n}^{n, n}(\approx) b_{0}+\overline{\bar{\delta}}_{n}^{n, n}(\approx) c_{0} \\
& =(-1)^{n-1}\left\{b^{n} c_{0}\right\}+(-1)^{n-1}\left\{P_{n-4}^{1} \hat{\bar{T}}_{4}^{n, n}+P_{n-4}^{2} \hat{\bar{T}}^{n, n}+P_{n-4}^{3} \hat{\bar{T}}_{2}^{n, n}+P_{n-4}^{4} \hat{\bar{T}}_{1}^{n, n}\right\} b_{0} \\
& +(-1)^{n-1}\left\{P_{n-5}^{1}\left(\hat{T}_{5}^{n, n} a_{0}+\hat{\overline{\bar{T}}}_{5}^{n, n} c_{0}\right)+P_{n-5}^{2}\left(\hat{T}_{4}^{n, n} a_{0}+\hat{\overline{\bar{T}}}_{4}^{n, n} c_{0}\right)\right. \\
& \left.+P_{n-5}^{3}\left(\hat{T}_{3}^{n, n} a_{0}+\hat{\overline{\bar{T}}}_{3}^{n, n} c_{0}\right)+P_{n-5}^{4}\left(\hat{T}_{2}^{n, n} a_{0}+\hat{\overline{\bar{T}}}_{2}^{n, n} c_{0}\right)\right\},
\end{aligned}
$$

where the initial values for $\hat{T}_{j}^{n, n}, \hat{\bar{T}}_{j}^{n, n}, \hat{\overline{\bar{T}}}_{j}^{n, n}$, are:

$$
\begin{aligned}
\hat{T}_{1}^{n, n} & =0 ; \quad \hat{T}_{2}^{n, n}=a_{0} r ; \quad \hat{T}_{3}^{n, n}=-a_{0} r-3 \sigma a_{0} r-b a_{0} r+2 \sigma r b_{0} ; \\
\hat{T}_{4}^{n, n} & =\left\{-\left(a_{0} r\right)-4 \sigma\left(a_{0} r\right)-b\left(a_{0} r\right)-7 \sigma^{2}\left(a_{0} r\right)-3 \sigma b\left(a_{0} r\right)-b^{2}\left(a_{0} r\right)\right. \\
& \left.-4 \sigma\left(a_{0} r\right) r+\left(a_{0} r\right) a_{0}^{2}+3 \sigma\left(a_{0} r\right) c_{0}+6\left(\sigma r b_{0}\right)+6 \sigma\left(\sigma r b_{0}\right)+2 b\left(\sigma r b_{0}\right)\right\} ; \\
\hat{T}_{5}^{n, n}= & \left\{-\left(a_{0} r\right)-5 \sigma\left(a_{0} r\right)-b\left(a_{0} r\right)-11 \sigma^{2}\left(a_{0} r\right)-4 \sigma b\left(a_{0} r\right)-b^{2}\left(a_{0} r\right)-15 \sigma^{3}\left(a_{0} r\right)\right. \\
& -7 \sigma^{2} b\left(a_{0} r\right)-3 \sigma b^{2}\left(a_{0} r\right)-b^{3}\left(a_{0} r\right)-12 \sigma\left(a_{0} r\right) r-20 \sigma \sigma\left(a_{0} r\right) r-4 b \sigma\left(a_{0} r\right) r \\
& +2\left(a_{0} r\right) a_{0}^{2}+10 \sigma\left(a_{0} r\right) a_{0}^{2}+2 b\left(a_{0} r\right) a_{0}^{2}+10 \sigma\left(a_{0} r\right) c_{0}+14 \sigma \sigma\left(a_{0} r\right) c_{0} \\
& +7 b \sigma\left(a_{0} r\right) c_{0}-13 \sigma\left(a_{0} r\right) a_{0} b_{0}+14\left(\sigma r b_{0}\right)+20 \sigma\left(\sigma r b_{0}\right)+6 b\left(\sigma r b_{0}\right) \\
& \left.+14 \sigma^{2}\left(\sigma r b_{0}\right)+6 \sigma b\left(\sigma r b_{0}\right)+2 b^{2}\left(\sigma r b_{0}\right)+8 \sigma\left(\sigma r b_{0}\right) r-4 \sigma\left(\sigma r b_{0}\right) c_{0}\right\} ; \\
\hat{\bar{T}}_{1}^{n, n} & =a_{0} ; \quad \hat{\bar{T}}_{2}^{n, n}=a_{0}+\sigma a_{0}+b a_{0}-\sigma b_{0} ;
\end{aligned}
$$




$$
\begin{aligned}
\hat{\bar{T}}_{3}^{n, n} & =\left\{a_{0}+2 \sigma a_{0}+b a_{0}+\sigma^{2} a_{0}+\sigma b a_{0}+b^{2} a_{0}+2 \sigma r a_{0}-a_{0} a_{0}^{2}-\sigma c_{0} a_{0}\right. \\
& \left.-3\left(\sigma b_{0}\right)-\sigma\left(\sigma b_{0}\right)-b\left(\sigma b_{0}\right)\right\} ; \\
\hat{\bar{T}}_{4}^{n, n} & =\left\{a_{0}+3 \sigma a_{0}+b a_{0}+3 \sigma^{2} a_{0}+2 \sigma b a_{0}+b^{2} a_{0}+\sigma^{3} a_{0}+\sigma^{2} b a_{0}+\sigma b^{2} a_{0}\right. \\
& +b^{3} a_{0}+6 \sigma a_{0} r+6 \sigma \sigma r a_{0}+2 \sigma b r a_{0}-2 a_{0} a_{0}^{2}-6 \sigma a_{0} a_{0}^{2}-2 b a_{0} a_{0}^{2} \\
& -4 \sigma a_{0} c_{0}-2 \sigma \sigma a_{0} c_{0}-2 \sigma b a_{0} c_{0}+7 a_{0} \sigma a_{0} b_{0}-7 \sigma b_{0}-4 \sigma\left(\sigma b_{0}\right) \\
& \left.-3 b\left(\sigma b_{0}\right)-\sigma^{2}\left(\sigma b_{0}\right)-\sigma b\left(\sigma b_{0}\right)-b^{2}\left(\sigma b_{0}\right)-4 \sigma r\left(\sigma b_{0}\right)+\sigma c_{0}\left(\sigma b_{0}\right)\right\} ; \\
\hat{\overline{\bar{T}}}_{1}^{n, n}= & 0 ; \quad \hat{\overline{\bar{T}}}^{n, n}=a_{0}^{2} ; \quad \hat{\bar{T}}_{3}^{n, n}=a_{0}^{2}+3 \sigma a_{0}^{2}+2 b a_{0}^{2}-3 \sigma a_{0} b_{0} ; \\
\hat{\overline{\bar{T}}}_{4}^{n, n} & =\left\{a_{0}^{2}+4 \sigma a_{0}^{2}+2 b a_{0}^{2}+7 \sigma^{2} a_{0}^{2}+8 \sigma b a_{0}^{2}+3 b^{2} a_{0}^{2}+5 \sigma r a_{0}^{2}-a_{0}^{2} a_{0}^{2}\right. \\
& \left.-4 \sigma c_{0} a_{0}^{2}-8 \sigma a_{0} b_{0}-10 \sigma\left(\sigma a_{0} b_{0}\right)-8 b\left(\sigma a_{0} b_{0}\right)+3 \sigma^{2} b_{0}^{2}\right\} ; \\
\hat{\bar{T}}_{5}^{n, n} & =\left\{a_{0}^{2}+5 \sigma a_{0}^{2}+2 b a_{0}^{2}+11 \sigma^{2} a_{0}^{2}+10 \sigma b a_{0}^{2}+3 b^{2} a_{0}^{2}+15 \sigma^{3} a_{0}^{2}+24 \sigma^{2} b a_{0}^{2}\right. \\
& +15 \sigma b^{2} a_{0}^{2}+4 b^{3} a_{0}^{2}+14 \sigma r a_{0}^{2}+26 \sigma \sigma r a_{0}^{2}+15 b \sigma r a_{0}^{2}-2 a_{0}^{2} a_{0}^{2}-10 \sigma a_{0}^{2} a_{0}^{2} \\
& -3 b a_{0}^{2} a_{0}^{2}-12 \sigma c_{0} a_{0}^{2}-20 \sigma \sigma c_{0} a_{0}^{2}-18 b \sigma c_{0} a_{0}^{2}+15 \sigma a_{0} b_{0} a_{0}^{2}-17 \sigma a_{0} b_{0} \\
& -30 \sigma\left(\sigma a_{0} b_{0}\right)-23 b\left(\sigma a_{0} b_{0}\right)-25 \sigma^{2}\left(\sigma a_{0} b_{0}\right)-35 \sigma b\left(\sigma a_{0} b_{0}\right)-15 b^{2}\left(\sigma a_{0} b_{0}\right) \\
& \left.-20 \sigma r\left(\sigma a_{0} b_{0}\right)+15 \sigma\left(\sigma a_{0} b_{0}\right) c_{0}+14 \sigma^{2} b_{0}^{2}+10 \sigma\left(\sigma^{2} b_{0}^{2}\right)+11 b\left(\sigma^{2} b_{0}^{2}\right)\right\} .
\end{aligned}
$$

For $j \in\{1,2,3,4,5\}$ we calculate from (1.3) the exact values of $c_{j}$ and we set $c_{j}(\approx)=c_{j}$ for $j \in\{0,1,2,3,4,5\}$.

\section{THE COEFFICIENT $b_{n}$}

For the coefficient $b_{n}$ we write the system (1.3) in the form:

$$
\begin{aligned}
& a_{n}=\sigma\left(b_{n-1}-a_{n-1}\right) \\
& b_{n}=\left(r-c_{0}\right) a_{n-1}-b_{n-1}-\sum_{i=0}^{n-2}\left(\begin{array}{c}
n-1 \\
i
\end{array}\right) a_{i} c_{n-1-i} \\
& c_{n}=b_{0} a_{n-1}-b c_{n-1}+\sum_{i=0}^{n-2}\left(\begin{array}{c}
n-1 \\
i
\end{array}\right) a_{i} b_{n-1-i}
\end{aligned}
$$

and for a fixed $n \in N$ and any $1 \leq k \leq n$ we represent $b_{n}$ as: 


$$
\begin{aligned}
b_{n} & =\bar{\varphi}_{k}^{n} a_{n-k}+\bar{\psi}_{k}^{n} b_{n-k}+\bar{\xi}_{k}^{n} c_{n-k}+\sum_{m=0}^{k-1} \sum_{i=k-m}^{n-k-1} \bar{\tau}_{k}^{n}(i, m) a_{i} b_{n-i-m-1} \\
& +\sum_{m=0}^{k-1} \sum_{i=k-m}^{n-k-1} \bar{\pi}_{k}^{n}(i, m) a_{i} c_{n-i-m-1}
\end{aligned}
$$

where $\bar{\varphi}_{k}^{n}, \bar{\psi}_{k}^{n}, \bar{\xi}_{k}^{n}, \bar{\tau}_{k}^{n}(i . m), \bar{\pi}_{k}^{n}(i, m)$ are new variables. For the new variables we obtain the new systems of difference equations:

$$
\begin{aligned}
& \bar{\varphi}_{k}^{n}=-\sigma \bar{\varphi}_{k-1}^{n}+\left(r-c_{0}\right) \bar{\psi}_{k-1}^{n}-\sum_{i=1}^{k-1}\left(\begin{array}{c}
n-k+i \\
n-k
\end{array}\right) c_{i} \bar{\psi}_{k-1-i}^{n} \\
& \bar{\psi}_{k}^{n}=\sigma \bar{\varphi}_{k-1}^{n}-\bar{\psi}_{k-1}^{n} \quad \bar{\xi}_{k}^{n}=0 \\
& \bar{\tau}_{k}^{n}(i, m)=0 \quad \bar{\pi}_{k}^{n}(i, m)=-\bar{\psi}_{k}^{n}\left(\begin{array}{c}
n-m-1 \\
i
\end{array}\right) .
\end{aligned}
$$

with $\bar{\varphi}_{0}^{n}=0, \bar{\psi}_{0}^{n}=1, \bar{\xi}_{0}^{n}=0$, and transform the presentation of $b_{n}$ to:

$$
b_{n}=\bar{\varphi}_{k}^{n} a_{n-k}+\bar{\psi}_{k}^{n} b_{n-k}-\sum_{m=0}^{k-1} \sum_{i=0}^{n-k-1}\left(\begin{array}{c}
n-1-m \\
i
\end{array}\right) \bar{\psi}_{m}^{n} a_{i} c_{n-i-m-1} .
$$

For $n=k$, the presentation of $b_{n}$ and the inequality $n-k-1<0$, imply:

4.1. $b_{n}=\bar{\varphi}_{n}^{n} a_{0}+\bar{\psi}_{n}^{n} b_{0}$.

Similarly as (2.3) and (2.4), for fixed $n, k \in N$ and any $1 \leq q \leq k$, we represent $\bar{\varphi}_{k}^{n}, \bar{\psi}_{k}^{n}$ as:

$$
\begin{aligned}
& \bar{\varphi}_{k}^{n}=\eta_{q}^{n, k} \bar{\varphi}_{k-q}^{n}+\omega_{q}^{n, k} \bar{\psi}_{k-q}^{n}+\sum_{m=0}^{q-1} \sum_{i=q-m}^{k-m-1} \kappa_{q}^{n, k}(i, m) c_{i} \\
& \bar{\psi}_{k}^{n}=\bar{\eta}_{q}^{n, k} \bar{\varphi}_{k-q}^{n}+\bar{\omega}_{q}^{n, k} \bar{\psi}_{k-q}^{n}+\sum_{m=0}^{q-1} \sum_{i=q-m}^{k-m-1} \bar{\kappa}_{q}^{n, k}(i, m) c_{i} .
\end{aligned}
$$

Again, similarly as $(2,6)$ and (2.7) we obtain the following systems:

$$
\begin{aligned}
& \eta_{q}^{n, k}=-\sigma \eta_{q-1}^{n, k}+\sigma \omega_{q-1}^{n, k} \\
& \omega_{q}^{n, k}=\left(r-c_{0}\right) \eta_{q-1}^{n, k}-\omega_{q-1}^{n, k}-\sum_{i=1}^{q-1} \eta_{q-1-i}^{n, k}\left(\begin{array}{c}
n-k+q-1 \\
i
\end{array}\right) c_{i} \\
& \kappa_{q}^{n, k}(i, m)=-\left(\begin{array}{c}
n-k+i+m \\
i
\end{array}\right) \eta_{m}^{n, k} \bar{\psi}_{k-m-i-1}^{n}
\end{aligned}
$$




$$
\begin{aligned}
& \bar{\eta}_{q}^{n, k}=-\sigma \bar{\eta}_{q-1}^{n, k}+\sigma \bar{\omega}_{q-1}^{n, k} \\
& \bar{\omega}_{q}^{n, k}=\left(r-c_{0}\right) \bar{\eta}_{q-1}^{n, k}-\bar{\omega}_{q-1}^{n, k}-\sum_{i=1}^{q-1} \bar{\eta}_{q-1-i}^{n, k}\left(\begin{array}{c}
n-k+q-1 \\
i
\end{array}\right) c_{i} \\
& \bar{\kappa}_{q}^{n, k}(i, m)=-\left(\begin{array}{c}
n-k+i+m \\
i
\end{array}\right) \bar{\eta}_{m}^{n, k} \bar{\psi}_{k-m-i-1}^{n}
\end{aligned}
$$

with initial values $\eta_{0}^{n, k}=1, \omega_{0}^{n, k}=0, \bar{\eta}_{0}^{n, k}=0, \bar{\omega}_{0}^{n, k}=1$.

For $q=k=n, \bar{\varphi}_{n}^{n}=\omega_{n}^{n, k}, \bar{\psi}_{n}^{n}=\bar{\omega}_{n}^{n, n}$, and by 4.1. we obtain:

4.2. $b_{n}=\omega_{n}^{n, n} a_{0}+\bar{\omega}_{n}^{n, n} b_{0}$.

By the same discussion as for the coefficient $a_{n}$ we arrived to the following presentations:

where:

$$
\omega_{q}^{n, k}=W_{q}^{n, k}+E_{q}^{n, k} \quad \bar{\omega}_{q}^{n, k}=\bar{W}_{q}^{n, k}+\bar{E}_{q}^{n, k}
$$

$$
\begin{aligned}
W_{q}^{n, k} & =(-1)^{q-1}\left[\sigma+\left(r-c_{0}\right)\right]^{q-1}\left(r-c_{0}\right) \\
& +(-1)^{q-1} \sum_{m=1}^{\left[\frac{q}{2}\right]} \sum_{j=m+1}^{q-m+1}\left(\begin{array}{c}
q-j \\
m-1
\end{array}\right)\left(\begin{array}{c}
j-1 \\
m-1
\end{array}\right) \sigma^{q-j}\left[\left(r-c_{0}\right)^{j}-\left(r-c_{0}\right)^{m}\right], \\
\bar{W}_{q}^{n, k} & =(-1)^{q}\left[\sigma+\left(r-c_{0}\right)\right]^{q-1}\left(r-c_{0}\right)+(-1)^{q-1}\left[\left(r-c_{0}\right)^{q}-1\right] \\
& +(-1)^{q-1} \sum_{m=2}^{\left[\frac{q+1}{2}\right.} \sum_{j=m}^{q-m+1}\left(\begin{array}{c}
q-j-1 \\
m-2
\end{array}\right)\left(\begin{array}{c}
j \\
m-1
\end{array}\right) \sigma^{q-j}\left[\left(r-c_{0}\right)^{j}-\left(r-c_{0}\right)^{m-1}\right],
\end{aligned}
$$

and $\hat{E}_{q}^{n, k}=(-1)^{q-1} E_{q}^{n, k}, \hat{\bar{E}}_{q}^{n, k}=(-1)^{q-1} \bar{E}_{q}^{n, k}$, have the form of the polynomials: $A \hat{E}_{q-1}^{n, k}+B \hat{E}_{q-2}^{n, k}+\bar{C} \hat{E}_{q-3}^{n, k}+D \hat{E}_{q-4}^{n, k}$ and $A \hat{\bar{E}}_{q-1}^{n, k}+B \hat{\bar{E}}_{q-2}^{n, k}+\bar{C} \hat{\bar{E}}_{q-3}^{n, k}+D \hat{\bar{E}}_{q-4}^{n, k}$, for: $A=1+\sigma+b, \quad B=\sigma\left(r-c_{0}\right)-a_{0}^{2}, \quad \bar{C}=\sigma a_{0} b_{0}-\sigma b c_{0}, \quad D=-\sigma^{2} b_{0}^{2}$.

So, for $q>5$ we approximate $\hat{E}_{q}^{n, n}$ with the solutions $\hat{E}_{q}^{n, n}(\approx)$ of the difference equation:

$$
\hat{E}_{q}^{n, n}(\approx)=A \hat{E}_{q-1}^{n, n}(\approx)+B \hat{E}_{q-2}^{n, n}(\approx)+\bar{C} \hat{E}_{q-3}^{n, n}(\approx)+D \hat{E}_{q-4}^{n, n}(\approx),
$$


with the initial values $\hat{E}_{2}^{n, n}, \hat{E}_{3}^{n, n}, \hat{E}_{4}^{n, n} \hat{E}_{5}^{n, n}$.

In the same way as for $a_{n}$, we take the presentation

$$
\hat{E}_{q}^{n, n}(\approx)=\bar{P}_{w}^{1} \hat{E}_{q-w}^{n, n}(\approx)+\bar{P}_{w}^{2} \hat{E}_{q-w-1}^{n, n}(\approx)+\bar{P}_{w}^{3} \hat{E}_{q-w-2}^{n, n}(\approx)+\bar{P}_{w}^{4} \hat{E}_{q-w-3}^{n, n}(\approx)
$$

and obtain the system of difference equations

$$
\begin{array}{ll}
\bar{P}_{w}^{1}=A \bar{P}_{w-1}^{1}+\bar{P}_{w-1}^{2} & \bar{P}_{w}^{2}=B \bar{P}_{w-1}^{1}+\bar{P}_{w-1}^{3}, \\
\bar{P}_{w}^{3}=\bar{C} P_{w-1}^{1}+\bar{P}_{w-1}^{4} & \bar{P}_{w}^{4}=D \bar{P}_{w-1}^{1}
\end{array},
$$

which is the same as the system (2.15) with $C$ replaced by $\bar{C}$. So, the solutions of (4.8) are the polynomials $\bar{P}_{w}^{1}, \bar{P}_{w}^{2}, \bar{P}_{w}^{3}, \bar{P}_{w}^{4}$ obtained from the polynomials $P_{w}^{1}, P_{w}^{2}, P_{w}^{3}, P_{w}^{4}$ with $C$ replaced by $\bar{C}$.

Similarly, for $q>6$ we approximate $\hat{\bar{E}}_{q}^{n, n}$ with the solutions $\hat{\bar{E}}_{q}^{n, n}(\approx)$ of the difference equation:

$$
\hat{\bar{E}}_{q}^{n, n}(\approx)=A \hat{\bar{E}}_{q-1}^{n, n}(\approx)+B \hat{\bar{E}}_{q-2}^{n, n}(\approx)+\bar{C} \hat{\bar{E}}_{q-3}^{n, n}(\approx)+D \hat{\bar{E}}_{q-4}^{n, n}(\approx),
$$

with the initial values $\hat{\bar{E}}_{3}^{n, n}, \hat{\bar{E}}_{4}^{n, n}, \hat{\bar{E}}_{5}^{n, n} \hat{\bar{E}}_{6}^{n, n}$.

Similarly as for $a_{n}$, all this produces the following approximation:

$$
\begin{aligned}
b_{n}(\approx) & =(-1)^{n-1}\left\{\bar{P}_{n-5}^{1} \hat{E}_{5}^{n, n}+\bar{P}_{n-5}^{2} \hat{E}_{4}^{n, n}+\bar{P}_{n-5}^{3} \hat{E}_{3}^{n, n}+\bar{P}_{n-5}^{4} \hat{E}_{2}^{n, n}\right\} a_{0}+W_{n}^{n, n} \\
& +(-1)^{n-1}\left\{\bar{P}_{n-6}^{1} \hat{\bar{E}}_{6}^{n, n}+\bar{P}_{n-6}^{2} \hat{\bar{E}}_{5}^{n, n}+\bar{P}_{n-6}^{3} \hat{\bar{E}}_{4}^{n, n}+\bar{P}_{n-6}^{4} \hat{\bar{E}}_{3}^{n, n}\right\} b_{0}+\bar{W}_{n}^{n, n}
\end{aligned}
$$

where the initial values for $\hat{E}_{j}^{n, n}$ and $\hat{\bar{E}}_{j}^{n, n}$ are:

$$
\begin{aligned}
\hat{E}_{1}^{n, n} & =0 ; \quad \hat{E}_{2}^{n, n}=-b c_{0}+a_{0} b_{0} ; \\
\hat{E}_{3}^{n, n} & =\left\{2\left(a_{0} b_{0}\right)+3 \sigma\left(a_{0} b_{0}\right)+b\left(a_{0} b_{0}\right)-\left(b c_{0}\right)-2 \sigma\left(b c_{0}\right)-b\left(b c_{0}\right)-a_{0}^{2}\left(r-c_{0}\right)-\sigma b_{0}^{2}\right\} ; \\
\hat{\bar{E}}_{1}^{n, n} & =0 ; \quad \hat{\bar{E}}_{2}^{n, n}=0 ; \quad \hat{\bar{E}}_{3}^{n, n}=-2 \sigma\left(a_{0} b_{0}\right)+2 \sigma\left(b c_{0}\right) ; \\
\hat{E}_{4}^{n, n} & =\left\{3\left(a_{0} b_{0}\right)+8 \sigma\left(a_{0} b_{0}\right)+2 b\left(a_{0} b_{0}\right)+7 \sigma^{2}\left(a_{0} b_{0}\right)+4 \sigma b\left(a_{0} b_{0}\right)+b^{2}\left(a_{0} b_{0}\right)\right. \\
& +8 \sigma\left(a_{0} b_{0}\right)\left(r-c_{0}\right)-\left(a_{0} b_{0}\right) a_{0}^{2}-\left(b c_{0}\right)-2 \sigma\left(b c_{0}\right)-b\left(b c_{0}\right)-3 \sigma^{2}\left(b c_{0}\right) \\
& -3 \sigma b\left(b c_{0}\right)-b^{2}\left(b c_{0}\right)-4 \sigma\left(b c_{0}\right)\left(r-c_{0}\right)+\left(b c_{0}\right) a_{0}^{2}-2 a_{0}^{2}\left(r-c_{0}\right) \\
& \left.-6 \sigma a_{0}^{2}\left(r-c_{0}\right)-b a_{0}^{2}\left(r-c_{0}\right)-4\left(\sigma b_{0}^{2}\right)-4 \sigma\left(\sigma b_{0}^{2}\right)-b\left(\sigma b_{0}^{2}\right)\right\} ;
\end{aligned}
$$




$$
\begin{aligned}
& \hat{\bar{E}}_{4}^{n, n}=\left\{-8\left(\sigma a_{0} b_{0}\right)-6 \sigma\left(\sigma a_{0} b_{0}\right)-3 b\left(\sigma a_{0} b_{0}\right)+5\left(\sigma b c_{0}\right)+3 \sigma\left(\sigma b c_{0}\right)+3 b\left(\sigma b c_{0}\right)\right. \\
& \left.+3 \sigma a_{0}^{2}\left(r-c_{0}\right)+3\left(\sigma^{2} b_{0}^{2}\right)\right\} \text {; } \\
& \hat{E}_{5}^{n, n}=\left\{4\left(a_{0} b_{0}\right)+15 \sigma\left(a_{0} b_{0}\right)+3 b\left(a_{0} b_{0}\right)+24 \sigma^{2}\left(a_{0} b_{0}\right)+10 \sigma b\left(a_{0} b_{0}\right)+2 b^{2}\left(a_{0} b_{0}\right)\right. \\
& +15 \sigma^{3}\left(a_{0} b_{0}\right)+11 \sigma^{2} b\left(a_{0} b_{0}\right)+5 \sigma b^{2}\left(a_{0} b_{0}\right)+b^{3}\left(a_{0} b_{0}\right)+32 \sigma\left(a_{0} b_{0}\right)\left(r-c_{0}\right) \\
& +46 \sigma \sigma\left(a_{0} b_{0}\right)\left(r-c_{0}\right)+11 b \sigma\left(a_{0} b_{0}\right)\left(r-c_{0}\right)-3\left(a_{0} b_{0}\right) a_{0}^{2}-10 \sigma\left(a_{0} b_{0}\right) a_{0}^{2} \\
& -2 b\left(a_{0} b_{0}\right) a_{0}^{2}-14 \sigma b\left(a_{0} b_{0}\right) c_{0}+11 \sigma\left(a_{0} b_{0}\right)^{2}-\left(b c_{0}\right)-2 \sigma\left(b c_{0}\right)-b\left(b c_{0}\right) \\
& -3 \sigma^{2}\left(b c_{0}\right)-3 \sigma b\left(b c_{0}\right)-b^{2}\left(b c_{0}\right)-4 \sigma^{3}\left(b c_{0}\right)-6 \sigma^{2} b\left(b c_{0}\right)-4 \sigma b^{2}\left(b c_{0}\right) \\
& -b^{3}\left(b c_{0}\right)-9 \sigma\left(b c_{0}\right)\left(r-c_{0}\right)-11 \sigma \sigma\left(b c_{0}\right)\left(r-c_{0}\right)-7 b \sigma\left(b c_{0}\right)\left(r-c_{0}\right) \\
& +2\left(b c_{0}\right) a_{0}^{2}+9 \sigma\left(b c_{0}\right) a_{0}^{2}+2 b\left(b c_{0}\right) a_{0}^{2}+4\left(\sigma b c_{0}\right)^{2}-3 a_{0}^{2}\left(r-c_{0}\right) \\
& -14 \sigma a_{0}^{2}\left(r-c_{0}\right)-2 b a_{0}^{2}\left(r-c_{0}\right)-25 \sigma^{2} a_{0}^{2}\left(r-c_{0}\right)-7 \sigma b a_{0}^{2}\left(r-c_{0}\right) \\
& -b^{2} a_{0}^{2}\left(r-c_{0}\right)-11 \sigma a_{0}^{2}\left(r-c_{0}\right)^{2}+a_{0}^{4}\left(r-c_{0}\right)-11\left(\sigma b_{0}^{2}\right)-20 \sigma\left(\sigma b_{0}^{2}\right) \\
& \left.-4 b\left(\sigma b_{0}^{2}\right)-11 \sigma^{2}\left(\sigma b_{0}^{2}\right)-5 \sigma b\left(\sigma b_{0}^{2}\right)-b^{2}\left(\sigma b_{0}^{2}\right)-11 \sigma\left(\sigma b_{0}^{2}\right)\left(r-c_{0}\right)\right\} \text {; } \\
& \hat{\bar{E}}_{5}^{n, n}=\left\{-22\left(\sigma a_{0} b_{0}\right)-30 \sigma\left(\sigma a_{0} b_{0}\right)-13 b\left(\sigma a_{0} b_{0}\right)-14 \sigma^{2}\left(\sigma a_{0} b_{0}\right)-10 \sigma b\left(\sigma a_{0} b_{0}\right)\right. \\
& -4 b^{2}\left(\sigma a_{0} b_{0}\right)-22 \sigma\left(\sigma a_{0} b_{0}\right)\left(r-c_{0}\right)+4\left(\sigma a_{0} b_{0}\right) a_{0}^{2}+9\left(\sigma b c_{0}\right)+7 \sigma\left(\sigma b c_{0}\right) \\
& +9 b\left(\sigma b c_{0}\right)+4 \sigma^{2}\left(\sigma b c_{0}\right)+6 \sigma b\left(\sigma b c_{0}\right)+4 b^{2}\left(\sigma b c_{0}\right)+6 \sigma\left(\sigma b c_{0}\right)\left(r-c_{0}\right) \\
& -4\left(\sigma b c_{0}\right) a_{0}^{2}+13 \sigma a_{0}^{2}\left(r-c_{0}\right)+18 \sigma \sigma a_{0}^{2}\left(r-c_{0}\right)+4 b \sigma a_{0}^{2}\left(r-c_{0}\right) \\
& \left.+21\left(\sigma^{2} b_{0}^{2}\right)+10 \sigma\left(\sigma^{2} b_{0}^{2}\right)+4 b\left(\sigma^{2} b_{0}^{2}\right)\right\} \text {; } \\
& \hat{\bar{E}}_{6}^{n, n}=\left\{-52\left(\sigma a_{0} b_{0}\right)-100 \sigma\left(\sigma a_{0} b_{0}\right)-38 b\left(\sigma a_{0} b_{0}\right)-84 \sigma^{2}\left(\sigma a_{0} b_{0}\right)\right. \\
& -50 \sigma b\left(\sigma a_{0} b_{0}\right)-19 b^{2}\left(\sigma a_{0} b_{0}\right)-30 \sigma^{3}\left(\sigma a_{0} b_{0}\right)-25 \sigma^{2} b\left(\sigma a_{0} b_{0}\right) \\
& -15 \sigma b^{2}\left(\sigma a_{0} b_{0}\right)-5 b^{3}\left(\sigma a_{0} b_{0}\right)-150 \sigma\left(\sigma a_{0} b_{0}\right)\left(r-c_{0}\right) \\
& -128 \sigma^{2}\left(\sigma a_{0} b_{0}\right)\left(r-c_{0}\right)-33 \sigma b\left(\sigma a_{0} b_{0}\right)\left(r-c_{0}\right)+24\left(\sigma a_{0} b_{0}\right) a_{0}^{2} \\
& +40 \sigma\left(\sigma a_{0} b_{0}\right) a_{0}^{2}+10 b\left(\sigma a_{0} b_{0}\right) a_{0}^{2}-45 \sigma a_{0} b_{0} \sigma a_{0} b_{0}+50 \sigma a_{0} b_{0} \sigma b c_{0} \\
& +14\left(\sigma b c_{0}\right)+12 \sigma\left(\sigma b c_{0}\right)+19 b\left(\sigma b c_{0}\right)+9 \sigma^{2}\left(\sigma b c_{0}\right)+16 \sigma b\left(\sigma b c_{0}\right) \\
& +14 b^{2}\left(\sigma b c_{0}\right)+5 \sigma^{3}\left(\sigma b c_{0}\right)+10 \sigma^{2} b\left(\sigma b c_{0}\right)+10 \sigma b^{2}\left(\sigma b c_{0}\right)+5 b^{3}\left(\sigma b c_{0}\right) \\
& +21 \sigma\left(\sigma b c_{0}\right)\left(r-c_{0}\right)+15 \sigma^{2}\left(\sigma b c_{0}\right)\left(r-c_{0}\right)+13 \sigma b\left(\sigma b c_{0}\right)\left(r-c_{0}\right)
\end{aligned}
$$




$$
\begin{aligned}
& -19\left(\sigma b c_{0}\right) a_{0}^{2}-35 \sigma\left(\sigma b c_{0}\right) a_{0}^{2}-10 b\left(\sigma b c_{0}\right) a_{0}^{2}-10 \sigma b c_{0} \sigma b c_{0}+96\left(\sigma^{2} b_{0}^{2}\right) \\
& +80 \sigma\left(\sigma^{2} b_{0}^{2}\right)+29 b\left(\sigma^{2} b_{0}^{2}\right)+25 \sigma^{2}\left(\sigma^{2} b_{0}^{2}\right)+15 \sigma b\left(\sigma^{2} b_{0}^{2}\right)+5 b^{2}\left(\sigma^{2} b_{0}^{2}\right) \\
& +33 \sigma\left(\sigma^{2} b_{0}^{2}\right)\left(r-c_{0}\right)+38 \sigma a_{0}^{2}\left(r-c_{0}\right)+88 \sigma \sigma a_{0}^{2}\left(r-c_{0}\right)+19 b \sigma a_{0}^{2}\left(r-c_{0}\right) \\
& +75 \sigma^{2} \sigma a_{0}^{2}\left(r-c_{0}\right)+25 \sigma b \sigma a_{0}^{2}\left(r-c_{0}\right)+5 b^{2} \sigma a_{0}^{2}\left(r-c_{0}\right) \\
& \left.+33 \sigma \sigma a_{0}^{2}\left(r-c_{0}\right)\left(r-c_{0}\right)-5 \sigma a_{0}^{4}\left(r-c_{0}\right)\right\} .
\end{aligned}
$$

For $j \in\{1,2,3,4,5,6\}$ we calculate from (1.3) the exact values of $b_{j}$ and we set $b_{j}(\approx)=b_{j}$ fro $j \in\{0,1,2,3,4,5,6\}$.

\section{LOCAL APPROXIMATIONS}

For parameters $\sigma, r, b$. and initial values $a_{0}, b_{0}, c_{0}$ we have the following power series:

$$
\begin{aligned}
& a_{0}(\approx)+a_{1}(\approx) t+a_{2}(\approx) \frac{t^{2}}{2 !}+\ldots+a_{n}(\approx) \frac{t^{n}}{n !}+\ldots=\sum_{n=0}^{\infty} a_{n}(\approx) \frac{t^{n}}{n !} \\
& b_{0}(\approx)+b_{1}(\approx) t+b_{2}(\approx) \frac{t^{2}}{2 !}+\ldots+b_{n}(\approx) \frac{t^{n}}{n !}+\ldots=\sum_{n=0}^{\infty} b_{n}(\approx) \frac{t^{n}}{n !} \\
& c_{0}(\approx)+c_{1}(\approx) t+c_{2}(\approx) \frac{t^{2}}{2 !}+\ldots+c_{n}(\approx) \frac{t^{n}}{n !}+\ldots=\sum_{n=0}^{\infty} c_{n}(\approx) \frac{t^{n}}{n !} .
\end{aligned}
$$

At this moment the following question is open. series (5.1)

Question: What conditions would imply the convergence of the power

Next we turn our attention to the following polynomials, (5.2).

$$
\begin{aligned}
& P_{m}\left(a_{0}, b_{0}, c_{0}\right)(t)=a_{0}(\approx)+a_{1}(\approx) t+\ldots+a_{m}(\approx) \frac{t^{m}}{m !}=\sum_{n=0}^{m} a_{n}(\approx) \frac{t^{n}}{n !} \\
& Q_{m}\left(a_{0}, b_{0}, c_{0}\right)(t)=b_{0}(\approx)+b_{1}(\approx) t+\ldots+b_{m}(\approx) \frac{t^{m}}{m !}=\sum_{n=0}^{m} b_{n}(\approx) \frac{t^{n}}{n !} \\
& R_{m}\left(a_{0}, b_{0}, c_{0}\right)(t)=c_{0}(\approx)+c_{1}(\approx) t+\ldots+c_{m}(\approx) \frac{t^{m}}{m !}=\sum_{n=0}^{m} c_{n}(\approx) \frac{t^{n}}{n !} .
\end{aligned}
$$


Let $T$ be a positive real number, and $m$ be a positive integer. For parameters $\sigma, r, b$ and initial $a_{0}, b_{0}, c_{0}$ we define functions $x_{T}(t), y_{T}(t), z_{T}(t)$ for $t \in[0, \infty)$, as follows.

For $t \in[0, T], \quad x_{T}(t)=P_{m}\left(a_{0}, b_{0}, c_{0}\right)(t), \quad y_{T}(t)=Q_{m}\left(a_{0}, b_{0}, c_{0}\right)(t)$ and $z_{T}(t)=R_{m}\left(a_{0}, b_{0}, c_{0}\right)(t)$.

Next, continue by induction. Assume that $x_{T}(t), y_{T}(t), z_{T}(t)$ are defined for $t \in[0, k \cdot T]$. We define them for $t \in[0,(k+1) \cdot T]$ as follows. For $t \in[0, k \cdot T]$ they are already defined, and for $t \in[k \cdot T,(k+1) \cdot T]$ we define them by: $x_{T}(t)=P_{m}\left(u_{0}, v_{0}, w_{0}\right)(t-k T), y_{T}(t)=Q_{m}\left(u_{0}, v_{0}, w_{0}\right)(t-k T)$ and $z_{T}(t)=R_{m}\left(u_{0}, v_{0}, w_{0}\right)(t-k T), \quad$ where $\quad u_{0}=x_{T}(k T), \quad v_{0}=y_{T}(k T) \quad$ and $w_{0}=x_{T}(k T)$.

At this moment we do not have the answer to the question of how good approximations, modulo $T$ and $m$, are the functions $x_{T}(t), y_{T}(t), z_{T}(t)$ for the solutions of the Lorenz system (1.1).

In examples, by computer calculations, for small values of $T$, we obtain that the functions $x_{T}(t), y_{T}(t), z_{T}(t)$ are good approximations for the solutions of the system (1.1). We used the program Mathematica and compared the solutions obtained by the program Mathematica with the functions $x_{T}(t), y_{T}(t), z_{T}(t)$. Two of these examples are the following.

Example 1: Parameters: $\sigma=10, r=23, b=5$; initial values $a_{0}=-2$, $b_{0}=3, c_{0}=0 ; T=0,05 ; m=20 ;$ and the time interval $[0,6]$.

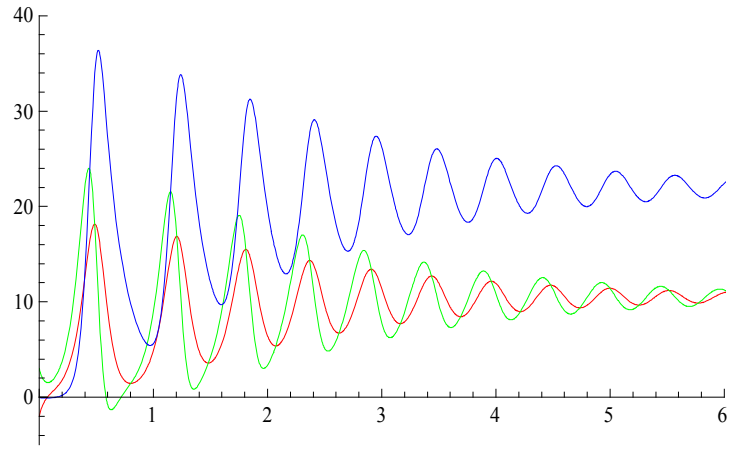

a) Graphics of the solutions

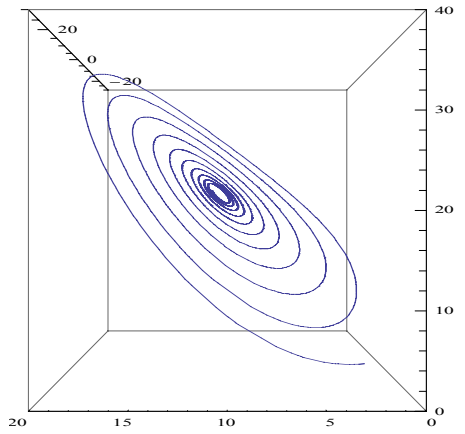

b) Space curve solution

Fig. 1. Results obtained by the program Mathematica 

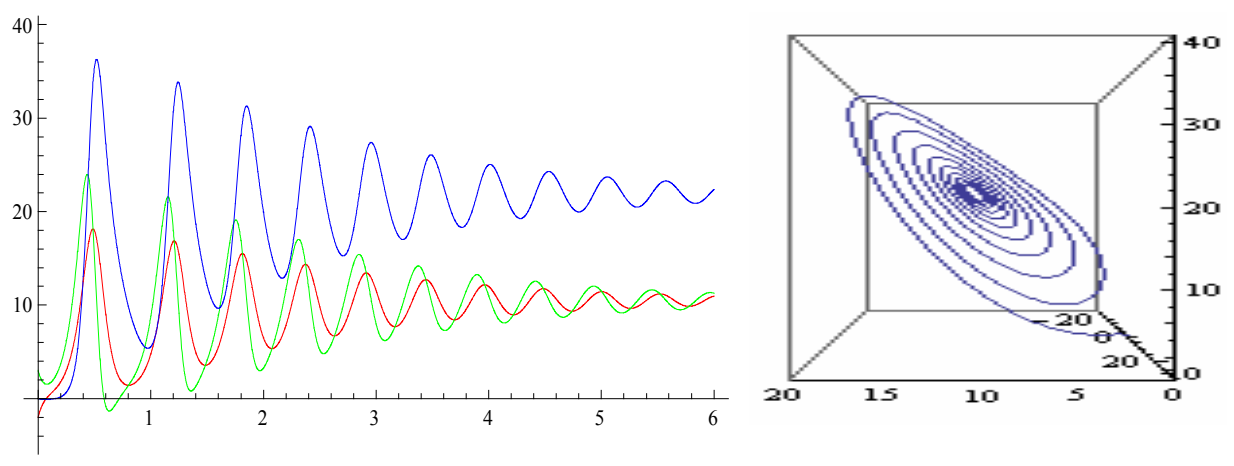

a) Graphics of the functions $x_{T}(t), y_{T}(t), z_{T}(t)$

b) Space curve $\left(x_{T}(t), y_{T}(t), z_{T}(t)\right)$

Fig. 2. Results obtained by computation

Example 2: Parameters: $\sigma=12, r=100, b=2$; initial values $a_{0}=32$, $b_{0}=20, c_{0}=50 ; T=0,05 ; m=20 ;$ and the time interval $[0,6]$.

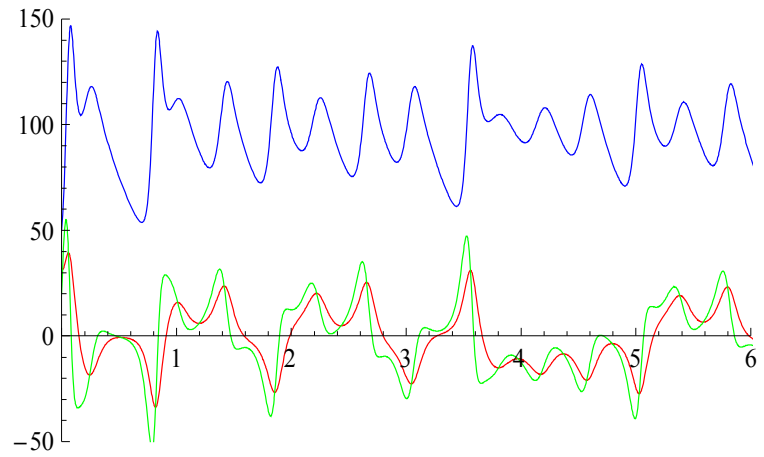

a) Graphics of the solutions

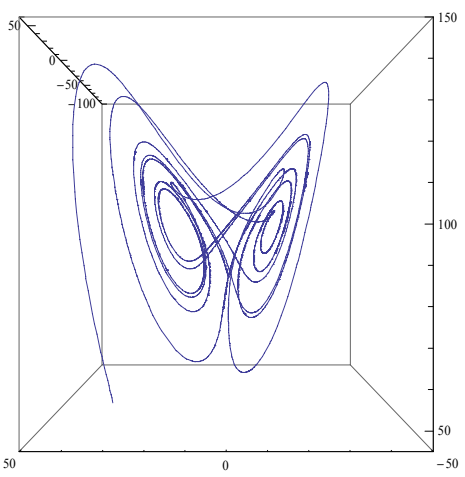

b) Space curve solution

Fig. 3. Results obtained by the program Mathematica

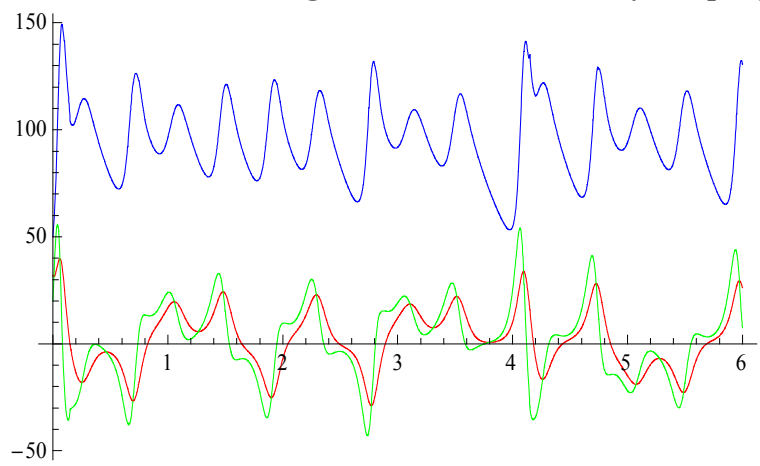

a) Graphics of the functions $x_{T}(t), y_{T}(t), z_{T}(t)$

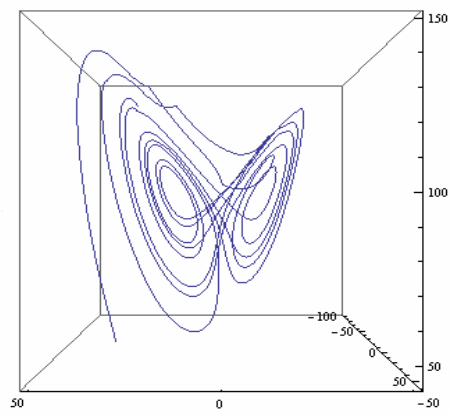

b) Space curve $\left(x_{T}(t), y_{T}(t), z_{T}(t)\right)$

Fig. 4. Results obtained by computation

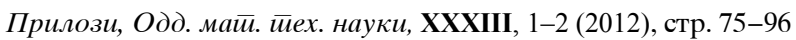




\section{REFERENCES}

[1] R. Barrio, F. Blesa, M. Lara, VSVO Formulation of the Taylor Method for the Numerical Solution of ODEs, Comput. Math. Appl., 50, (2005), 93-111.

[2] Barrio, R. Performance of the Taylor series method for ODEs/DAEs, Comput. Math. Appl., 163, (2005), 525-545.

[3] M. A. Fathi, An Analytical Solution for the Modified Lorenz System, Proceedings of the World Congress on Engineering 2012 (ISSN: 2078-0966 (Online)) Vol. I, London, U.K..

[4] M. W. Hirsch, S. Smale, R. L. Devaney, Differential Equations, Dynamical System and An Introduction to Chaos, USA, 2004.

[5] S. Lynch, Dynamical Systems with Applications using Mathematica, Boston, USA, 2007.

[6] Wiggins S. Introduction to applied nonlinear dynamical systems and chaos, Springer, 2003.

Р е 3 и м е

\section{СИСТЕМИ ОД ДИФЕРЕНЦНИ РАВЕНКИ КАКО АПРОКСИМАЦИЈА НА ЛОРЕНЦОВИОТ СИСТЕМ ОД ДИФЕРЕНЦИЈАЛНИ РАВЕНКИ}

Во овој труд од Лоренцовиот систем диференцијални равенки се добиени системи диференцни равенки. Користејќ некои регуларности во овие ситеми од диференцни равенки, добиени се полиномни апроксимации на нивните решенија. Земајќи ги овие апроксимации како коефициенти, добиени се три степенски реда и со компјутерски пресметки е проверувано дека тие даваат локална апроксимација на решенијата на Лоренцовиот систем диференцијални равенки.

\section{Клучни зборови: Лоренцов систем; диференцијални равенки; диференцни равенки; степенски редови; апроксимација}

Address:

Biljana Zlatanovska

University "Goce Delčev", Faculty of Computer Science

Štip,Republic of Macedonia

zlatanovskabiljana_1973@yahoo.com

\section{Dončo Dimovski}

Ss. Cyril and Methodius University in Skopje

Faculty of Natural Sciences and Mathematics, PO Box 162, MK-1001 Skopje, Republic of Macedonia donco@pmf.ukim.mk 